\title{
Consumer choice under limited attention when options have different information costs
}

Frank Huettner, ESMT European School of Management and Technology

Tamer Boyacı, ESMT European School of Management and Technology

Yalçın Akçay, College of Administrative Sciences and Economics, Koç University

Copyright 2016 by ESMT European School of Management and Technology GmbH, Berlin, Germany, www.esmt.org. All rights reserved. No part of this publication may be reproduced, stored in a retrieval system, used in a spreadsheet, or transmitted in any form or by any means - electronic, mechanical, photocopying, recording, or otherwise - without the permission of ESMT.

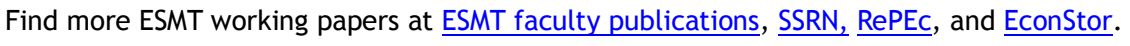




\title{
Consumer Choice Under Limited Attention When Options Have Different Information Costs
}

\author{
Frank Huettner, Tamer Boyacı \\ ESMT European School of Management and Technology, \\ Schlossplatz 1, 10178, Berlin, Germany \\ frank.huettner@esmt.org, tamer.boyaci@esmt.org \\ Yalçın Akçay \\ College of Administrative Sciences and Economics, Koç University, \\ Rumeli Feneri Yolu, Istanbul, 34450, Turkey \\ yakcay@ku.edu.tr
}

\begin{abstract}
Consumers often do not have complete information about the choices they face and therefore have to spend time and effort in acquiring information. Since information acquisition is costly, consumers have to trade-off the value of better information against its cost, and make their final choices based on imperfect information. We model this decision using the rational inattention approach and describe the rationally inattentive consumer's choice behavior when she faces options with different information costs. To this end, we introduce an information cost function that distinguishes between direct and inferential information. We then analytically characterize the optimal behavior and derive the choice probabilities in closed-form. We find that non-uniform information costs can have a strong impact on product choice, which gets particularly conspicuous when the product alternatives are otherwise very similar. It can also lead to situations where it is disadvantageous for the seller to provide easier access to information for a particular product. Furthermore, it provides a new explanation for strong failure of regularity of consumer behaviour, which occurs if the addition of an inferior - never chosen - product to the choice set increases the market share of another existing product.
\end{abstract}

Key words: discrete choice, rational inattention, information acquisition, non-uniform information costs, strong failure of regularity.

\section{Introduction}

Facing an abundance of product choices and related information, but with only limited time and attention to evaluate them, consumers have to come to grips with how much and what type of information to acquire and to pay attention to (and what to ignore), and make product choice and purchase decisions based on this partial information. Since information is "costly", consumers have 
to trade-off the value of better information against its cost. Rational inattention theory offers a compelling approach to capture this connection since it is built on the premise that attention is a scarce resource and therefore has to be allocated wisely. ${ }^{1}$ Specifically, the pioneering works of Sims $(1998,2003,2006)$ propose a framework in which utility-maximizing decision-makers acquire information optimally, where information is quantified as reduction in Shannon entropy. Based on a stream of literature in information theory, this approach works without specifying how decisionmakers acquire information or what they may learn. In other words, the information acquisition process is fully endogenized, and rationally inattentive decision-makers optimally select the type and quantity of information they need, and ignore the information that is not worth obtaining and processing. In a recent paper, Matějka and McKay (2015) show that when faced with discrete choices with stochastic (payoff) values, a rationally inattentive consumer's optimal information processing strategy leads to a choice behaviour that can be characterized as generalized multinomial logit (GMNL). In particular, the choice probabilities depend not only on the true realizations of the choices as in the standard MNL, but also on the prior beliefs of the consumer and the cost of information.

The GMNL model offers a micro-founded characterization of consumer choice under limited attention that is intuitively appealing as well. It does so, however, under an important assumption: the cost of information is uniform across all options. This can be interpreted as the consumer acquiring and processing information through a common channel with a certain associated cost. Effectively, it means that the amount of effort (and hence cost) spent to obtain and process 1-byte of information about each option is the same. Clearly, this is not necessarily the case in reality. It is oftentimes easier to obtain information about some products than about others, by the very nature of the product. Or sometimes information is obtained from different sources with different levels of timeand-attention-efficiency (online, catalog, direct salesforce etc). It can also depend on the assortment that is offered - it is easier to obtain information about products that are readily available for "touch and feel" compared to others that are not available for such an experience and require extra effort to garner information. ${ }^{2}$ These realities call for a choice model that allows the information cost to vary among different options considered by the consumer. In this paper, we generalize the GMNL model by describing the rationally inattentive consumer's choice behavior when she faces options with different information costs.

Quantifying the amount of information the consumer acquires when evaluating a particular option and accounting for its cost become more intricate in the presence of a non-uniform information cost

\footnotetext{
1 Throughout the paper we use the terms "limited attention" and "rational inattention" interchangeably.
}

${ }^{2}$ S. J. Hoch (1986) and Hamilton and Thompson (2007) stress the importance of consumers' experience at the point of sale and consumers' struggle to judge on the value of a product through abstract product description compared with direct user experience. 
Huettner, Boyacı, and Akçay: Consumer Choice When Options Have Different Information Costs

structure, and this gets even more pronounced when there are similarities (i.e., correlations) between the products. This is because, as the consumer learns about a product, she may also learn about another product. To exemplify this, suppose there are two products, Product 1 and 2. ProduCT 1 is available at a retail store the consumer is currently visiting, while PRODUCT 2 is not available there but sold elsewhere (e.g. online store) requiring extra travel/search effort for the consumer. The consumer knows that these products are very similar (in theory they can even be identical) and in line with the above description, assumes that the information cost is lower at the retail store. The consumer might inquire about PRODUCT 1 at the retail store, and this would also reveal significant information about PRODUCT 2. The consumer might make a decision based on this or go and visit the online store and acquire more information about PRODUCT 2 (she will decide on this optimally) and then make a choice. This example highlights the fact that there are two forms of information acquired by the consumer: (i) direct information that the consumer obtains by studying the particular option, and (ii) inferential information that the consumer acquires about one option by studying another option. Since the unit costs of these sources might differ, it becomes important to glean from the consumer's information processing strategy, the amount of information acquired from each source. In addition, it must also be that the rationally inattentive consumer is efficient in processing information, i.e., she should go to the cheapest source to obtain information. Picking up our example again, this means she should never inquire about PRODUCT 1 in the online store, and for PRoDuCT 2 she should inquire at the on-line store only to obtain information beyond the information that she can infer from Product 1 (already obtained at the retail store). The novel aspects of our model is the development of an information cost function that is consistent with the efficient processing principle, and that it quantifies separately the amount of inferential and direct information. Our approach generalizes the Shannon entropy based cost function utilized in the rational inattention literature.

We formulate and analyze the consumer's discrete choice problem, and then determine the structure of the optimal solution. We show that consumer's optimal choice behaviour can be characterized analytically in closed-form. This characterization generalizes the GMNL model in the sense that our choice model reduces to GMNL when the cost of information is uniform across all options. After rigorously establishing this result, we concentrate on a number of special limiting cases involving infinite information costs, zero information costs, duplicate options, with the additional objective of highlighting some features of the optimal choice. Subsequently, we focus on an auxiliary example, the classical red-bus/blue-bus problem, and illustrate the impact of multiple information channels and different costs on the choice behavior of consumers with limited attention.

The theory of rational inattention belongs to the literature of bounded rationality since the works of Simon $(1955,1979)$ and receives significant interest in economics (Gabaix 2014) and psychology 
(Todd and Gigerenzer 2000). Different from the consideration set approach (e.g. Manzini and Mariotti 2014), all options are kept on the table in rational inattention models. The consumer decides whether information about the characteristics of the products is processed or not. In Reis (2006) the consumer either pays a fee to become fully informed or not, while in other models the consumer may decide on the degree of the precision to which she receives information (e.g. in the models of Verrecchia (1982), the consumer decides on the variance of the signals). The models conceived by Sims (1998) and later adopted by many other researchers generalize this approach, as they offer the consumer the opportunity to receive signals of any type and to improve her prior in every desirable way. Our paper follows this stream of modeling rational inattention. The key is to model the cost of information as a reduction of uncertainty with respect to the prior, where uncertainty is measured as Shannon entropy (Shannon 1948). For a motivation of Shannon entropy as a measure of uncertainty, we refer the reader to the axiomatic treatment in Csiszár (2008) and to the excellent introduction by Cover and Thomas (2006). Early applications of models of rational inattention adopting this approach are largely found in the macroeconomics literature (Sims 2003, Maćkowiak and Wiederholt 2009). Since then the realm of studies involving rational inattention has expanded to include also microeconomics topics, especially pricing (e.g., Matějka and McKay 2012, Matějka 2015 and Boyacı and Akçay 2016).

The fact that customers need to exercise differing levels of effort in order to get informed about different alternatives has been studied for multiple purposes in the context of sequential search models. In these models, consumers gather information about a particular product one-by-one (and possibly one attribute at a time), and purchase once they decide to stop collecting more information optimally (e.g. Weitzman 1979, Branco et al. 2012, Ke et al. 2016). Our approach differs from these models in that we make no assumption on the process by which the consumer gets informed nor on the type or quantity of information acquired (the information strategy is fully endogenized).

Models of rational inattention are particularly promising because of their close relation to MNL models. We refer to Anderson et al. (1992) for a comprehensive coverage of MNL models in general and to Wierenga (2008) for their use in marketing science. MNL and its variations (e.g., nested logit) have been extensively used to model consumer behavior in the operations management literature as well, in particular in the context of pricing, revenue management and assortment planning (Hanson and Martin 1996, van Ryzin and Mahajan 1999, Dong et al. 2009, Zhang and Adelman 2009, Davis et al. 2014, among many others). In this stream, the need for richer and more general choice models has also been recognized and some propositions have been made, such as Talluri and van Ryzin (2004), Alptekinoğlu and Semple (2015), Blanchet et al. (2016), Srikanth and Rusmevichientong (2016). Our paper complements this literature by offering a new, general and versatile choice model that is derived from an analysis of the optimal behaviour of an individual consumer. 
Our contribution to the literature is three-fold. First, we add more realism to the characterization of choice by rationally inattentive consumers, effectively generalizing the GMNL. Second, utilizing our choice model, we provide insights regarding the impact of different information costs on the optimal choice of consumers. By comparison with the case of uniform costs, we show that the impact can indeed be quite substantial. Furthermore, the detailed assessment of information costs in our framework allows insight into the attention allocation strategy of the consumer, which drives the ultimate choice behavior. Finally, our choice characterization provides the essential building block for a variety of operations/marketing applications involving consumers with limited attention. Assortment planning constitutes such an example. The static assortment decision of a firm involves the selection of products to be offered to consumers. An accurate model for such a problem should capture the fact that products offered in the assortment are accessible to the consumers more directly and hence bear lower information costs than those products left outside the assortment.

\section{Choice Model Formulation}

In this section, we develop the choice model for a rationally inattentive consumer with different information costs across alternatives. The consumer can choose from the finite set of alternatives $\mathcal{A}=\{1, \ldots, n\}$. The state of nature is a random variable $\mathbf{V}$ taking values $\mathbf{v} \in \mathbb{R}^{n}$, where $v_{i}$ denotes the value of alternative $i \in \mathcal{A}$. The consumer has the prior belief $p \in \Delta\left(\mathbb{R}^{n}\right)$ where $\Delta\left(\mathbb{R}^{n}\right)$ denotes the set of all probability distributions on $\mathbb{R}^{n}$. In order to improve her decision, the consumer can process information with the goal of sharpening her belief about the state of nature. Let $\mathbf{S}=\mathbb{R}^{n}$ denote the signal space that is available to the consumer. Consistent with the theory of rational inattention, we assume that the consumer can set up any joint distribution $f_{\mathbf{S}, \mathbf{V}}$ of signals and values, given that it is consistent with her prior belief, i.e., the marginal of $f$ with respect to $\mathbf{V}$ equals $p$,

$$
f_{\mathbf{V}}(\mathbf{v})=\int_{\mathbf{s} \in \mathbb{R}^{n}} f_{\mathbf{S}, \mathbf{v}}(d \mathbf{s}, \mathbf{v})=p(\mathbf{v}) \quad \text { for all } \mathbf{v} \in \mathbb{R}^{n} .
$$

Clearly, depending on the joint distribution of signals and values, the signal can be more or less beneficial. In particular, given the reception of signal $\mathbf{s} \in \mathbb{R}^{n}$, the consumer creates an updated belief $f_{\mathbf{V} \mid \mathbf{s}}$ over the values of the options and chooses the alternative that gives the highest expected value under the updated belief, yielding the expected payoff

$$
U\left(f_{\mathbf{V} \mid \mathbf{s}}\right)=\max _{i \in \mathcal{A}} \mathbb{E}_{f_{\mathbf{V} \mid \mathbf{s}}}\left[v_{i}\right]
$$

The less noise that remains in the updated belief, the more promising this choice becomes. Signals that reveal the true state and eliminate all uncertainty allow the consumer to make a perfect choice. This would maximize the payoff

$$
R(f)=\int_{\mathbf{v} \in \mathbb{R}^{n}} \int_{\mathbf{s} \in \mathbb{R}^{n}} U\left(f_{\mathbf{V} \mid \mathbf{s}}\right) f_{\mathbf{S} \mid \mathbf{v}}(d \mathbf{s}) p(d \mathbf{v}) .
$$


The consumer has no restrictions in choosing her information strategy as long as it is consistent with her priors, but information processing is costly. Specifically, information costs depend on the extent of the reduction of uncertainty, measured by entropy $\mathcal{H}$. The a-priori entropy of the state of nature is given by

$$
\mathcal{H}(p)=\sum_{\mathbf{v} \in \mathbb{R}^{n}} p(\mathbf{v}) \cdot(-\log p(\mathbf{v})) .
$$

Receiving signal s yields a reduction of this entropy by $\mathcal{H}(p)-\mathcal{H}\left(f_{\mathbf{V} \mid \mathbf{s}}\right)$ to the level of the entropy of the posterior belief $f_{\mathbf{V} \mid \mathbf{s}}$. The expectation of this reduction over all signals is called mutual information between $\mathbf{S}$ and $\mathbf{V}$ under the joint distribution $f$, and is denoted by $\mathcal{I}_{f}(\mathbf{V}, \mathbf{S})$

$$
\mathcal{I}_{f}(\mathbf{V}, \mathbf{S})=\mathcal{H}(p)-\sum_{\mathbf{s} \in \mathbf{S}} f_{\mathbf{S}}(\mathbf{s}) \mathcal{H}\left(f_{\mathbf{V} \mid \mathbf{s}}\right)
$$

In particular, $\mathcal{I}_{f}(\mathbf{V}, \mathbf{S})$ quantifies the extent of what the consumer expects to learn about the state of nature from the signals.

Extant literature assumes that the cost per unit of mutual information $\lambda$ is uniform across all alternatives, and accordingly defines the total cost of information as $C(f)=\lambda \cdot \mathcal{I}_{f}(\mathbf{V}, \mathbf{S})$. Matějka and McKay (2015) show that the information strategy optimizing the net pay-off $R(f)-C(f)$ results in a choice behavior that can be characterized as Generalized Multinomial Logit (GMNL). Specifically, the conditional probability $p(i \mid \mathbf{v})$ of choosing alternative $i \in A$ when the state is $\mathbf{v}$ is given as

$$
p(i \mid \mathbf{v})=\frac{e^{\frac{v_{i}}{\lambda}} \cdot p(i)}{\sum_{j=1}^{n} e^{\frac{v_{j}}{\lambda}} \cdot p(j)}
$$

Here $p(i):=\int_{\mathbf{v} \in \mathbb{R}^{n}} p(i \mid \mathbf{v}) p(d \mathbf{v})$ represents the unconditional probability of selecting alternative $i \in \mathcal{A}$. Note that $p(i)$ are not exogenous parameters; they are part of the consumer's decision making strategy, capturing the effects of prior beliefs. When the consumer is a-priori indifferent to all alternatives (i.e., $p(\mathbf{v})$ is invariant to all permutations of the elements of $\mathbf{v}$ ), then $p(i)=\frac{1}{n}$ and (3) reduces to the standard MNL formula.

In this paper, we relax the uniform information cost assumption and let $\lambda_{i}$ denote the unit cost of acquiring information for alternative $i$. Without loss of generality, suppose that the alternatives are ordered such that $\lambda_{1} \leq \lambda_{2} \ldots \leq \lambda_{n}$. At this point, one could speculate that the resulting conditional choice probabilities should resemble (3) with $\lambda$ simply replaced with $\lambda_{i}$ for each alternative. This conjecture, however, turns out to be incorrect. As we show later, the conditional choice probabilities can still be characterized in closed-form similar to the GMNL formula, but the "attraction" term associated with each alternative is more involved.

The most crucial element of the rationally inattentive consumer's choice framework with nonuniform information costs is the development of a total information cost function that correctly 
accounts for the sources of information. As noted earlier, the cost function should distinguish the amount of inferential and direct information obtained for each alternative, and reflect the "efficiency" principle in obtaining this information (i.e., the customer should prioritize cheaper channels to acquire information). We next present the derivation of this total information function, which utilizes the conditional mutual information between the alternatives and the signals generated from the queries of the customers. In order to simplify the exposition, suppose that there are only two alternatives, Product 1 and Product 2, for which $\mathbf{V}=\left(V_{1}, V_{2}\right)$ with $0<\lambda_{1} \leq \lambda_{2}<\infty$. As ProdUCT 1 has a lower information cost, let us first focus on the information learned about PRODUCT 1 from the signals. To this end, let $p_{V_{1}}$ and $f_{V_{1} \mid \mathbf{s}}$ denote the marginals of the prior and the posterior with respect to the value of PRODUCT 1. By asking questions, the consumer receives signals that reduce the uncertainty of her knowledge of $V_{1}$. This reduction is the difference between the prior and posterior entropies, i.e., the mutual information between $\mathbf{S}$ and $V_{1}$,

$$
\mathcal{I}_{f}\left(V_{1}, \mathbf{S}\right)=\mathcal{H}\left(p_{V_{1}}\right)-\sum_{\mathbf{s} \in \mathbf{S}} f_{\mathbf{S}}(\mathbf{s}) \mathcal{H}\left(f_{V_{1} \mid \mathbf{s}}\right)
$$

Figure 1 illustrates this relationship on a Venn Diagram. The entropy of $V_{1}$ is reduced by $\mathcal{I}_{f}\left(V_{1}, \mathbf{S}\right)$, i.e., by the intersection of $\mathcal{H}\left(p_{V_{1}}\right)$ and $\mathcal{H}\left(f_{\mathbf{S}}\right)$. Note that in a similar vein, the mutual information $\mathcal{I}_{f}\left(V_{2}, \mathbf{S}\right)$ between $\mathbf{S}$ and $V_{2}$ is given by the intersection of $\mathcal{H}\left(p_{V_{2}}\right)$ and $\mathcal{H}\left(f_{\mathbf{S}}\right)$, while the mutual information $\mathcal{I}\left(V_{1}, V_{2}\right)$ between $V_{1}$ and $V_{2}$ is given by the intersection of $\mathcal{H}\left(p_{V_{1}}\right)$ and $\mathcal{H}\left(p_{V_{2}}\right)$.

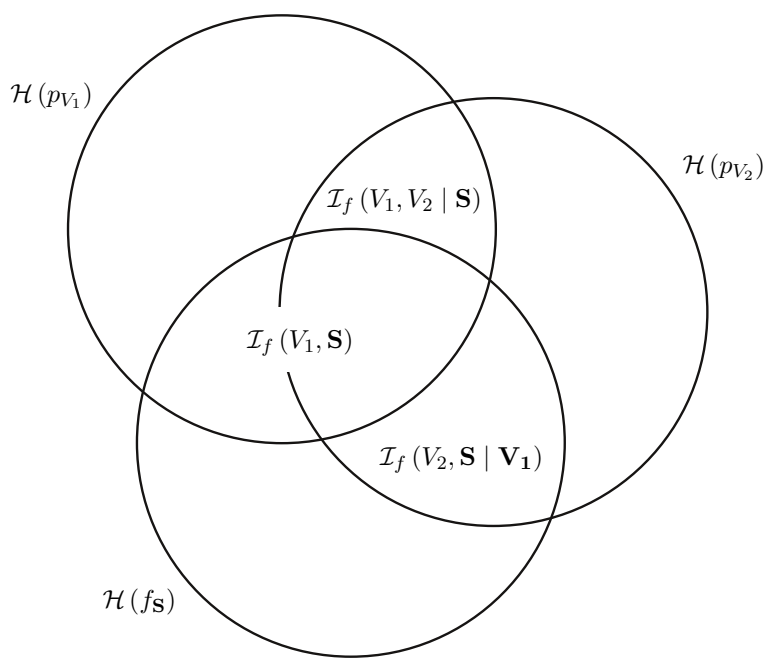

Figure 1 Venn diagram illustrating the relationship between entropy, mutual information and conditional mutual information

Since the signals that inform about PRODUCT 1 are processed through a channel that cost $\lambda_{1}$ per information unit ${ }^{3}$, the cost incurred by this activity is $\lambda_{1} \mathcal{I}_{f}\left(V_{1}, \mathbf{S}\right)$. Obviously, there is no ${ }^{3}$ When the base of the logarithm is 2 , this unit is bytes. 
reason for the consumer to learn about PRODUCT 1 indirectly from the more costly channel, so all of this is direct information. However, when the consumer processes information through this channel and learns about PRODUCT 1, she typically also learns about PRODUCT 2. In other words, a part of what is learned from the signal about PRODUCT 2 is already contained in the mutual information of the signal and PRODUCT 1. This inferential information about PRODUCT 2 can be quantified as the intersection of $\mathcal{I}_{f}\left(V_{1}, \mathbf{S}\right)$ and $\mathcal{I}_{f}\left(V_{2}, \mathbf{S}\right)$ in Figure 1. Clearly, the consumer should not attempt to acquire this information by directly inquiring about PRODUCT 2 - all direct information acquisition efforts should be concentrated on learning what she cannot infer via PRODUCT 1 . This additional information is the mutual information between $V_{2}$ and $\mathbf{S}$ conditional on $V_{1}, \mathcal{I}_{f}\left(V_{2}, \mathbf{S} \mid V_{1}\right)$. To quantify this information, we need to first determine the information learned about the entire state variable $\left(V_{1}, V_{2}\right)$ from the signal, i.e. $\mathcal{I}_{f}\left(V_{1}, V_{2} ; \mathbf{S}\right)$, which is given as the union of the intersection of $\mathcal{H}\left(p_{V_{1}}\right)$ and $\mathcal{H}\left(p_{V_{2}}\right)$ with $\mathcal{H}\left(f_{\mathbf{S}}\right)$. Subtracting from this from the information $\mathcal{I}_{f}\left(V_{1}, \mathbf{S}\right)$ that the consumer already knows from Product 1, we get the mutual information between $V_{2}$ and $\mathbf{S}$ conditional on $V_{1}$ :

$$
\mathcal{I}_{f}\left(V_{2}, \mathbf{S} \mid V_{1}\right)=\mathcal{I}_{f}\left(V_{1}, V_{2} ; \mathbf{S}\right)-\mathcal{I}_{f}\left(V_{1}, \mathbf{S}\right)
$$

This additional information is acquired at a unit cost $\lambda_{2}$, and the total cost of information becomes

$$
C(f)=\lambda_{1} \mathcal{I}_{f}\left(V_{1}, \mathbf{S}\right)+\lambda_{2} \mathcal{I}_{f}\left(V_{2}, \mathbf{S} \mid V_{1}\right)
$$

The above described process of accounting for information costs is consistent with the principle of processing information through the cheapest channel/source. It delineates inferential and direct information in accordance with this principle and charges the unit cost appropriately. With this total cost function, the consumer is able to optimize on her information channels and learn about each alternative up to an optimal extent.

The Venn diagram in Figure 1 aids in visualizing the relationship between mutual information and conditional mutual information among alternatives and signals and facilitates description of our cost function. It is important to recognize however that this relationship is more intricate than it may seem from the Venn diagram, even for the case with two alternatives. For example, consider the intersection of $\mathcal{H}\left(p_{V_{1}}\right), \mathcal{H}\left(p_{V_{2}}\right)$ and $\mathcal{H}\left(f_{\mathbf{S}}\right)$. This area, which is referred to as the mutual information of three variables (as in Cover and Thomas 2006) or interaction information (as in McGill 1954) can actually represent a negative quantity. Hence, it is possible that $\mathcal{I}_{f}\left(V_{2}, \mathbf{S} \mid V_{1}\right)>\mathcal{I}_{f}\left(V_{2}, \mathbf{S}\right)$. To see this more lucidly, consider the case where $V_{1}$ and $V_{2}$ are independent. Then, there is no mutual information between $V_{1}$ and $V_{2}$, i.e., $\mathcal{I}\left(V_{1}, V_{2}\right)=0$, and knowing $V_{1}$ does not reveal anything about $V_{2}$. It seems quite plausible then that $\mathcal{I}_{f}\left(V_{2}, \mathbf{S} \mid V_{1}\right)=\mathcal{I}_{f}\left(V_{2}, \mathbf{S}\right)$. However, this is not true. Even though $V_{1}$ and $V_{2}$ are a-priori independent, they do "become" dependent because of the signals 
received. That is, $V_{1}$ and $V_{2}$ are dependent conditional on $\mathbf{S}$ such that the mutual information of $V_{1}$ and $V_{2}$ conditional on $\mathbf{S}, \mathcal{I}_{f}\left(V_{1}, V_{2} \mid \mathbf{S}\right)$, is non-zero ${ }^{4}$. Observe that from the symmetry of mutual information, $\mathcal{I}_{f}\left(V_{1}, V_{2} \mid \mathbf{S}\right)$ can be written as (which can be retraced in Figure 1)

$$
\mathcal{I}_{f}\left(V_{2}, \mathbf{S} \mid V_{1}\right)=\mathcal{I}_{f}\left(V_{2}, \mathbf{S}\right)+\mathcal{I}_{f}\left(V_{1}, V_{2} \mid \mathbf{S}\right)-\mathcal{I}\left(V_{1}, V_{2}\right)
$$

Hence, even though $\mathcal{I}\left(V_{1}, V_{2}\right)=0$, it is the case that $\mathcal{I}_{f}\left(V_{2}, \mathbf{S} \mid V_{1}\right)>\mathcal{I}_{f}\left(V_{2}, \mathbf{S}\right)$.

Returning to our derivation, one can easily see that the construction with two alternative products given in (4) readily extends to the more general case with $n$ alternatives, by defining the total cost of information as

$$
C(f)=\sum_{i \in \mathcal{A}} \lambda_{i} \cdot \mathcal{I}_{f}\left(V_{i}, \mathbf{S} \mid \mathbf{V}_{1 \cdots i-1}\right)
$$

where $\mathbf{V}_{1 \cdot i-1}$ denotes $\left(V_{1}, \ldots, V_{i-1}\right)$ and the mutual information $\mathcal{I}_{f}\left(V_{i}, \mathbf{S} \mid \mathbf{V}_{1 \cdot i-1}\right)$ is given recursively by

$$
\mathcal{I}_{f}\left(V_{i}, \mathbf{S} \mid \mathbf{V}_{1 \cdots i-1}\right)=\mathcal{I}_{f}\left(\mathbf{V}_{1 \cdot i}, \mathbf{S}\right)-\sum_{k=1}^{i-1} \mathcal{I}_{f}\left(V_{k}, \mathbf{S} \mid \mathbf{V}_{1 \cdots k-1}\right) .
$$

Note that $\lambda_{i}=\lambda$ for all $i \in \mathcal{A}$ implies $C(f)=\lambda \cdot \mathcal{I}_{f}(\mathbf{V}, \mathbf{S})$ and our model reduces to that in the existing literature.

As a result, the rationally inattentive consumer's discrete choice strategy is dictated by her information strategy $f$ which solves

$$
\begin{aligned}
& \max _{f \in \Delta\left(\mathbb{R}^{2 n}\right)} R(f)-C(f) \\
& \text { s.t. } \quad \int_{\mathbf{s} \in \mathbb{R}^{n}} f_{\mathbf{S}}(d \mathbf{s}, \mathbf{v})=f(\mathbf{v}) \quad \text { for all } \mathbf{v} \in \mathbb{R}^{n}
\end{aligned}
$$

where $R(f)$ and $C(f)$ are given in equations (2) and (7), respectively. Here it is implicit that the consumer makes the optimal choice based on her posterior belief. In case the consumer is indifferent between two alternatives, she uses an arbitrary rule to break any such ties.

\section{Optimal Choice}

In this section, we solve the optimization model given in (9)-(10), and describe the ensuing optimal choice probabilities. To this end, we follow the standard approach in rational inattention literature. We first show that an optimal information strategy would generate only one posterior belief for each alternative. In other words, if an information strategy were to lead to the choice of a particular alternative under distinct posteriors, the consumer would have processed "unnecessary" information, and hence such a strategy would not be optimal. This enables us to restate the consumer's problem

\footnotetext{
${ }^{4}$ This can be formalized via a contradiction argument.
} 
in terms of conditional choice probabilities directly. Solving this problem yields the characterization of the optimal choice behavior.

For a given information strategy $f$, we define the set of signals that lead to the choice of alternative $i$ as

$$
\begin{aligned}
S_{i}(f):= & \left\{\mathbf{s} \in \mathbb{R}^{n} \mid i \in \arg \max _{i \in \mathcal{A}} \mathbb{E}_{f_{\mathbf{V} \mid \mathbf{s}}}\left[v_{i}\right]\right. \\
& \text { and } \left.i \text { is chosen according to the tie-breaking rule if \# } \arg \max _{i \in \mathcal{A}} \mathbb{E}_{f_{\mathbf{V} \mid \mathbf{s}}}\left[v_{i}\right]>1 .\right\}
\end{aligned}
$$

Accordingly, we calculate the conditional choice probability for alternative $i$ given state $\mathbf{v}$ as

$$
p_{f}(i \mid \mathbf{v}):=\int_{\mathbf{s} \in S_{i}} f_{\mathbf{S} \mid \mathbf{v}}(d \mathbf{s}),
$$

and the unconditional probability of choosing $i$ as

$$
p_{f}(i):=\int_{\mathbf{v} \in \mathbb{R}^{n}} p_{f}(i \mid \mathbf{v}) p(d \mathbf{v}) .
$$

Suppose that two distinct signals $\mathbf{s}^{\prime}$ and $\mathbf{s}^{\prime \prime}$ result in the choice of the same alternative $i$, i.e., $\mathbf{s}^{\prime}, \mathbf{s}^{\prime \prime} \in S_{i}$, with different posteriors under an optimal information strategy $f^{*}$, i.e., $f_{\mathbf{V} \mid \mathbf{s}^{\prime}}^{*} \neq f_{\mathbf{V} \mid \mathbf{s}^{\prime \prime}}^{*}$. Now consider another information strategy, say $\tilde{f}$, which is identical to $f^{*}$ for all $\mathbf{s} \notin\left\{\mathbf{s}^{\prime}, \mathbf{s}^{\prime \prime}\right\}$. Under $\tilde{f}$, however, the consumer receives signal $\mathbf{s}^{\prime}$ whenever she would have received either $\mathbf{s}^{\prime}$ or $\mathbf{s}^{\prime \prime}$ under $f^{*}$. Note that if the consumer chooses alternative $i$ with strategy $f^{*}$, she would also make the same choice with $\tilde{f}$. In Lemma 1 , we formally prove that such an $f^{*}$ cannot be an optimal information strategy, since $\tilde{f}$ pays off at least as much as $f^{*}$, i.e., $R(\tilde{f}) \geq R\left(f^{*}\right)$, and costs less, i.e., $C(\tilde{f}) \leq C\left(f^{*}\right)$.

Lemma 1. Let $f^{*}$ be optimal and let $i \in A$ be such that $p_{f^{*}}(i \mid \mathbf{v})>0$. Then, for all signals $\mathbf{s}^{\prime}, \mathbf{s}^{\prime \prime} \in$ $S_{i}$ the posterior beliefs are identical, i.e., $f_{\mathbf{V} \mid \mathbf{s}^{\prime}}^{*}=f_{\mathbf{V} \mid \mathbf{s}^{\prime \prime}}^{*}$, up to a set of measure zero under $f^{*}$.

Lemma 1 suggests that the consumer processes information efficiently under the optimal strategy, and essentially needs only a single signal per choice alternative. Based on this result, we can express the consumer's objective given in (9) directly as a function of the choice probabilities, without referring to the signal space $\mathbf{S}$ and other intricate details of the information strategy $f$. Particularly, we focus our attention on those strategies with $n$ distinct signals $s_{1}, \ldots, s_{n}{ }^{5}$. Since receiving signal $s_{i}$ is equivalent to choosing alternative $i$, i.e., $f_{\mathbf{S} \mid \mathbf{v}}\left(s_{i}\right)=p_{f}(i \mid \mathbf{v})$, the payoff $R(f)$, previously defined in (2), can now be expressed as

$$
R(f)=\sum_{s_{i} \in \mathbf{S}} U\left(f_{\mathbf{V} \mid s_{i}}\right) \cdot \int_{\mathbf{v} \in \mathbb{R}^{n}} p_{f}(i \mid \mathbf{v}) p(d \mathbf{v})=\sum_{s_{i} \in \mathbf{S}} \int_{\mathbf{v} \in \mathbb{R}^{n}} v_{i} f_{\mathbf{V} \mid s_{i}}(\mathbf{v}) d \mathbf{v} \cdot p_{f}(i)
$$

\footnotetext{
${ }^{5}$ The exact argument does allow for multiple signals leading to $i,\left|S_{i}\right| \geq 1$, and is given in the appendix.
} 
Note that the term $p_{f}(i) \cdot f_{\mathbf{V} \mid s_{i}}(\mathbf{v})$ specifies the joint probability that the state of nature $\mathbf{V}$ assumes the value $\mathbf{v}$ and that the consumer chooses alternative $i$. Hence, we also have $p_{f}(i) \cdot f_{\mathbf{V} \mid s_{i}}(\mathbf{v})=$ $p_{f}(i \mid \mathbf{v}) \cdot p(\mathbf{v})$. Then, we can rewrite $R(f)$ as just a function of the conditional choice probabilities

$$
R(f)=\sum_{i \in \mathcal{A}} \int_{\mathbf{v} \in \mathbb{R}^{n}} v_{i} p_{f}(i \mid \mathbf{v}) p(d \mathbf{v}) .
$$

Similarly, we can restate the cost $C(f)$ given in $(7)$ as

$$
C(f)=\sum_{i \in A} \lambda_{i} \cdot \mathcal{I}_{f}\left(V_{i}, \mathbf{A} \mid \mathbf{V}_{1 \cdots i-1}\right)
$$

where $\mathbf{A}$ denotes the random variable that takes value $i \in \mathcal{A}$ with probability $p_{f}(i)$. Here, we once again recognize that the consumer's choice behaviour, captured by the random variable $\mathbf{A}$, is as informative about the state of nature $\mathbf{V}$, as the signal space $\mathbf{S}$ leading to the consumer's choices, i.e., $\mathcal{I}_{f}\left(V_{i}, \mathbf{S} \mid \mathbf{V}_{1 \cdot i-1}\right)=\mathcal{I}_{f}\left(V_{i}, \mathbf{A} \mid \mathbf{V}_{1 \cdot i-1}\right)$. As a result, using (11) and (12), we can express the objective function (9) directly as a function of the choice probabilities without making any implicit reference to the information strategy (even in the form of a subscript). The next proposition presents the resulting formulation to the consumer's choice problem.

Proposition 1. The set of conditional choice probabilities $\{p(i \mid \mathbf{v})\}_{i \in \mathcal{A}}$ leads to the optimal information strategy of an inattentive consumer (optimally solves the problem in (9)-(10)), if and only if it is a solution to the following problem

$$
\max _{\{p(i \mid \mathbf{v})\}_{i \in \mathcal{A}}} \sum_{i \in \mathcal{A}} \int_{\mathbf{v} \in \mathbb{R}^{n}} v_{i} p(i \mid \mathbf{v}) p(d \mathbf{v})-\sum_{j \in \mathcal{A}} \lambda_{j} \cdot \mathcal{I}_{p}\left(V_{j}, \mathbf{A} \mid \mathbf{V}_{1 \cdot \cdot j-1}\right)
$$

subject to

$$
\begin{aligned}
p(i \mid \mathbf{v}) & \geq 0 \quad \text { for all } i \in \mathcal{A} \text { and } \mathbf{v} \in \mathbb{R}^{n} \\
\sum_{i \in \mathcal{A}} p(i \mid \mathbf{v}) & =1
\end{aligned}
$$

where

$$
\mathcal{I}_{p}\left(V_{j}, \mathbf{A} \mid \mathbf{V}_{1 \cdot . j-1}\right)=-\sum_{i \in \mathcal{A}} \int_{\mathbf{v}_{1 \cdot . j} \in \mathbb{R}^{j}} p\left(i \mid \mathbf{v}_{1 \cdot . j}\right)\left(\log p\left(i \mid \mathbf{v}_{1 \cdot . j-1}\right)-\log p\left(i \mid \mathbf{v}_{1 \cdot . j}\right)\right) p\left(d \mathbf{v}_{1 \cdot . j}\right)
$$

and

$$
p\left(i \mid \mathbf{v}_{1 \cdot \cdot j}\right)=\int_{\mathbf{v}_{j+1 \cdot \ell \ell} \in \mathbb{R}^{n-j}} p\left(i \mid \mathbf{v}_{1 \cdot . j} \mathbf{v}_{j+1 \cdots n}\right) p\left(\mathbf{v}_{j+1 \cdot \cdots n} \mid \mathbf{v}_{1 \cdot \cdot j}\right) d \mathbf{v}_{j+1 \cdot n} .
$$

Next, we consider how one can go about solving this alternative formulation given in Proposition 1. First note that this formulation is a concave maximization problem on a compact set. The objective function in (13) is concave in $\{p(i \mid \mathbf{v})\}_{i \in \mathcal{A}}$, since $\mathcal{I}_{p}\left(V_{j}, \mathbf{A} \mid \mathbf{V}_{1 \cdot . j-1}\right)=\mathcal{D}\left(p\left(i, \mathbf{v}_{1 \cdot . j}\right) \| p\left(i, \mathbf{v}_{1 \cdot . j-1}\right)\right.$. 
$\left.p\left(\mathbf{v}_{1 . . j}\right)\right)$ is convex in $\{p(i \mid \mathbf{v})\}_{i \in \mathcal{A}}$. Here, $\mathcal{D}$ denotes the relative entropy, which is convex in the pair $\left(p\left(i, \mathbf{v}_{1 . . j}\right), p\left(i, \mathbf{v}_{1 \cdot . j-1}\right) \cdot p\left(\mathbf{v}_{1 \cdot . j}\right)\right)$ and hence in $p(i, \mathbf{v})$ (c.f. Cover and Thomas 2006, Theorem 2.7.2). Moreover, as we show in the Appendix, $p\left(i \mid \mathbf{v}_{1 . . j}\right)=0$ only if $p(i)=0$. Treating this case separately, the optimization problem essentially has one equality constraint, which allows us to obtain the structure of the optimal solution from the first order conditions of the Lagrangian. We present this result next in Theorem 1.

TheOREM 1. For any information cost structure $0<\lambda_{1} \leq \lambda_{2} \leq \ldots \leq \lambda_{n}<\infty$, the consumer forms her information strategy such that the optimal conditional choice probabilities satisfy

$$
p(i \mid \mathbf{v})=\frac{e^{\frac{v_{i}}{\lambda_{n}}} \cdot p(i)^{\frac{\lambda_{1}}{\lambda_{n}}} \cdot \prod_{k=1}^{n-1} p\left(i \mid \mathbf{v}_{1 . . k}\right)^{\frac{\lambda_{k+1}-\lambda_{k}}{\lambda_{n}}}}{\sum_{j=1}^{n} e^{\frac{v_{j}}{\lambda_{n}}} \cdot p(j)^{\frac{\lambda_{1}}{\lambda_{n}}} \cdot \prod_{k=1}^{n-1} p\left(j \mid \mathbf{v}_{1 . . k}\right)^{\frac{\lambda_{k+1}-\lambda_{k}}{\lambda_{n}}}}
$$

almost surely.

We remark that (15) is derived for alternatives with a positive probability of being chosen, i.e., $p(i)>0$, but it trivially holds for alternatives never chosen, i.e., $p(i)=0$, as well. Notice also that the conditional choice probability in (15) resembles the GMNL formula - in fact, when information costs are identical, (16) becomes the exact GMNL expression in (3). It is more general in the sense that when information costs differ, the conditional choice probabilities are not only driven by state $\mathbf{v}$, prior beliefs $p(\mathbf{v})$ and unconditional probabilities $p(i)$, but also by the partially conditional choice probabilities $p\left(j \mid \mathbf{v}_{1 . . k}\right)$ 's of selecting each alternative. To gain a better understanding of the implications, it is useful to rewrite (15) as

$$
p(i \mid \mathbf{v})=\frac{e^{\frac{v_{i}}{\lambda_{n}}+\alpha_{i}}}{\sum_{j \in \mathcal{A}} e^{\frac{v_{j}}{\lambda_{n}}+\alpha_{j}}},
$$

where we define $\alpha_{i}$ as

$$
\alpha_{i}=\frac{\lambda_{1}}{\lambda_{n}} \cdot \log p(i)+\sum_{k=1}^{n-1} \frac{\lambda_{k+1}-\lambda_{k}}{\lambda_{n}} \cdot \log p\left(i \mid \mathbf{v}_{1 . . k}\right) .
$$

Written this way, the conditional probabilities follow a formula similar to the standard MNL, with the pay-off of each alternative shifted by the term $\alpha_{i}$. For the GMNL, $\alpha_{i}$ simply equals $\log p(i)$, implying that if an alternative is in general attractive, i.e., $p(i)$ is relatively high, it can still be chosen with high probability even if its true value is low (Matějka and McKay 2015). When the information costs are different, the consumer will typically know more about the cheaper options, and this is reflected into the computation of how "attractive" the alternative is. Specifically, the shift term is a weighted average of the log transformations of the unconditional and partially conditional choice probabilities. Hence, a generally attractive alternative (with a relatively high $p(i)$ ) can be 
chosen with a low probability if the information obtained from studying an alternative with low cost (say Alternative 1) implies a low selection probability $p\left(i \mid \mathbf{v}_{1}\right)$, even if the true value is high.

The next proposition restates the consumer's choice problem in Proposition 1 using the structure of the conditional choice probabilities presented in Theorem 1 .

Proposition 2. The set of conditional choice probabilities $\left\{p\left(i \mid \mathbf{v}_{1 \cdot n-1}\right)\right\}_{i \in \mathcal{A}}$ solves the problem in Proposition 1 if and only if it is a solution to the following problem

$$
\begin{aligned}
& \max _{\left\{p\left(i \mid \mathbf{v}_{1 \cdot n-1}\right)\right\}_{i \in \mathcal{A}}} \lambda_{n} \int_{\mathbf{v}} \log \left(\sum_{j=1}^{n} e^{\frac{v_{j}}{\lambda_{n}}} \cdot p(j)^{\frac{\lambda_{1}}{\lambda_{n}}} \cdot \prod_{k=1}^{n-1} p\left(j \mid \mathbf{v}_{1 \cdot . k}\right)^{\frac{\lambda_{k+1}-\lambda_{k}}{\lambda_{n}}}\right) p(d \mathbf{v}) \quad \text { s.t. } \\
& p\left(i \mid \mathbf{v}_{1 \cdot n-1}\right) \geq 0 \quad \text { for all } i \in \mathcal{A} \text { and } \mathbf{v}_{1 \cdot n-1} \in \mathbb{R}^{n-1}, \\
& \sum_{i \in \mathcal{A}} p\left(i \mid \mathbf{v}_{1 \cdot n-1}\right)=1 .
\end{aligned}
$$

We should remark that the formulation in Proposition 2 offers a significant simplification over the initial formulation in (9)-(10) for finding the optimal information strategy. Nevertheless, solving for the optimal $\left\{p\left(i \mid \mathbf{v}_{1 \cdot n-1}\right)\right\}_{i \in \mathcal{A}}$ can still be quite challenging when there are many alternatives and possible realizations of the values. Therefore, in what follows, we first examine some limiting cases and then present some illustrative examples to better understand the inattentive consumer's choice behavior facing alternatives with non-uniform information costs.

\section{Limiting Scenarios and Choice Behavior}

In the previous section, we characterized the optimal behaviour of customers for the most general case involving distinct alternatives with non-zero and finite information costs. There are some limiting scenarios that do not directly follow from the conditional choice probability equation (15) in Theorem 1. In this section, we focus on four such scenarios - infinite and zero information cost for some alternatives, and duplicate and dominated alternatives. Delving into these limiting cases also sheds some light on how non-uniform information costs impact the choices of inattentive consumers. To keep the exposition simple, we assume that the consumer chooses one of three alternative products - Product 1, Product 2, and Product 3 - with information costs of $\lambda_{1} \leq \lambda_{2} \leq \lambda_{3}$, respectively (we provide the formulae for the general case in Appendix B). The conditional choice probabilities for these three alternatives can be expressed as:

$$
p\left(i \mid v_{1}, v_{2}, v_{3}\right)=\frac{e^{\frac{v_{i}}{\lambda_{3}}} \cdot p(i)^{\frac{\lambda_{1}}{\lambda_{3}}} \cdot p\left(i \mid v_{1}\right)^{\frac{\lambda_{2}-\lambda_{1}}{\lambda_{3}}} \cdot p\left(i \mid v_{1}, v_{2}\right)^{\frac{\lambda_{3}-\lambda_{2}}{\lambda_{3}}}}{\sum_{j \in\{1,2,3\}} e^{\frac{v_{j}}{\lambda_{3}}} \cdot p(j)^{\frac{\lambda_{1}}{\lambda_{3}}} \cdot p\left(j \mid v_{1}\right)^{\frac{\lambda_{2}-\lambda_{1}}{\lambda_{3}}} \cdot p\left(j \mid v_{1}, v_{2}\right)^{\frac{\lambda_{3}-\lambda_{2}}{\lambda_{2}}}}
$$

for all $i \in \mathcal{A}=\{1,2,3\}$ and all $\mathbf{v}=\left(v_{1}, v_{2}, v_{3}\right) \in \mathbb{R}^{3}$. 
INFINITE INFORMATION COST. Suppose that it is infinitely costly (or prohibitively expensive) for the consumer to process information about PRODUCT 3. This could represent a product for which the customer is not willing to acquire any information, or for which such information is not obtainable (e.g. product is not offered/available). Since, $\lambda_{3}=\infty$ and

$$
C(f)=\lambda_{1} \mathcal{I}_{f}\left(V_{1}, \mathbf{S}\right)+\lambda_{2} \mathcal{I}_{f}\left(V_{2}, \mathbf{S} \mid V_{1}\right)+\lambda_{3} \mathcal{I}_{f}\left(V_{3}, \mathbf{S} \mid V_{1}, V_{2}\right)
$$

the consumer would have to set $\mathcal{I}_{f}\left(V_{3}, \mathbf{S} \mid V_{1}, V_{2}\right)=0$ under her optimal information strategy (to avoid an infinite information processing cost). Accordingly, the consumer does not update her priors beyond the information about Product 2. Then, from (14),

$$
\begin{aligned}
\mathcal{I}_{f}\left(V_{3}, \mathbf{S} \mid V_{1}, V_{2}\right)=0 & \Rightarrow \log p\left(i \mid v_{1}, v_{2}\right)-\log p\left(i \mid v_{1}, v_{2}, v_{3}\right)=0 \\
& \Rightarrow p\left(i \mid v_{1}, v_{2}, v_{3}\right)=p\left(i \mid v_{1}, v_{2}\right) .
\end{aligned}
$$

The above expression implies that the conditional choice probabilities do not depend on $v_{3}$. However this does not mean that she makes decisions about PRODUCT 3 based on prior beliefs only. In fact, the customer updates her expectation of the value $v_{3}$ on the basis of information learned about Product 1 and Product 2 values $\left(v_{1}, v_{2}\right)$. Defining this conditional expectation as $\bar{v}_{3}\left(v_{1}, v_{2}\right)=$ $\mathbb{E}_{p_{V_{3} \mid v_{1}, v_{2}}}\left[v_{3}\right]$, the conditional choice probabilities in (17) can be rewritten as

$$
p\left(i \mid v_{1}, v_{2}, v_{3}\right)=\frac{q\left(i \mid v_{1}, v_{2}\right)}{\sum_{j \in\{1,2,3\}} q\left(j \mid v_{1}, v_{2}\right)} \quad \text { for all } i \in\{1,2,3\},
$$

where

$$
\begin{aligned}
& q\left(1 \mid v_{1}, v_{2}\right)=e^{\frac{v_{1}}{\lambda_{2}}} \cdot p(1)^{\frac{\lambda_{1}}{\lambda_{2}}} \cdot p\left(1 \mid v_{1}\right)^{\frac{\lambda_{2}-\lambda_{1}}{\lambda_{2}}}, \\
& q\left(2 \mid v_{1}, v_{2}\right)=e^{\frac{v_{2}}{\lambda_{2}}} \cdot p(2)^{\frac{\lambda_{1}}{\lambda_{2}}} \cdot p\left(2 \mid v_{1}\right)^{\frac{\lambda_{2}-\lambda_{1}}{\lambda_{2}}}, \\
& q\left(3 \mid v_{1}, v_{2}\right)=e^{\frac{\bar{v}_{3}\left(v_{1}, v_{2}\right)}{\lambda_{2}}} \cdot p(3)^{\frac{\lambda_{1}}{\lambda_{2}}} \cdot p\left(3 \mid v_{1}\right)^{\frac{\lambda_{2}-\lambda_{1}}{\lambda_{2}}} .
\end{aligned}
$$

Zero Information Cost (and Deterministic Alternatives). Suppose that the consumer can freely process all information for PRODUCT 1, i.e, $\lambda_{1}=0$. This could represent a product for which the customer can assign a true value very easily (e.g. a simple search good). Then, taking the limit of (17) with $\lambda_{1} \rightarrow 0$, we get

$$
p\left(i \mid v_{1}, v_{2}, v_{3}\right)=\frac{e^{\frac{v_{i}}{\lambda_{3}}} \cdot p\left(i \mid v_{1}\right)^{\frac{\lambda_{2}}{\lambda_{3}}} \cdot p\left(i \mid v_{1}, v_{2}\right)^{\frac{\lambda_{3}-\lambda_{2}}{\lambda_{3}}}}{\sum_{j \in\{1,2,3\}} e^{\frac{v_{j}}{\lambda_{3}}} \cdot p\left(j \mid v_{1}\right)^{\frac{\lambda_{2}}{\lambda_{3}}} \cdot p\left(j \mid v_{1}, v_{2}\right)^{\frac{\lambda_{3}-\lambda_{2}}{\lambda_{3}}}} \quad \text { for all } i \in\{1,2,3\} .
$$

Note that if the value of ProduCT 1 is deterministic, i.e., $V_{1}=v_{1}$ with probability 1 , then $p(i)=p\left(i \mid v_{1}\right)$ even for $\lambda_{1}>0$. Accordingly, (17) also reduces to the above expression for deterministic alternatives. This could represent a product about which the consumer is well-informed due 
to past experience, or the no-purchase option (reservation value). We remark however that only the functional form of the conditional choice probabilities are the same; the actual values of the conditional probabilities and resulting behavior are likely to differ. This is because learning about a deterministic alternative reveals no information about other alternatives. In contrast learning the exact value of an alternative can reveal significant information about other alternatives. More precisely, for a deterministic alternative, $V_{1}$ takes only one value, whereas alternatives with zero information cost can potentially take an arbitrary number of values. Hence the partially conditional purchase probabilities involving $V_{1}$ (e.g. $p\left(i \mid v_{1}, v_{2}\right)$ ) would average over all these potential values.

Next, we provide an example involving the above limiting scenarios to demonstrate the impact on consumer choice.

Example 1 The consumer is choosing from Product 1, Product 2 and Product 3. There are 3 possible states, and let $\mathbf{v} \in\left\{(1,0,0),(0,1,0),\left(0,0, \frac{1}{2}\right)\right\}$ and $p(\mathbf{v})=\frac{1}{3}$ for all $\mathbf{v}$. Table 1 depicts the unconditional choice probability of choosing each alternative for different levels of information costs.

Table 1 Information costs and unconditional choice probabilities

\begin{tabular}{|c|ccc||ccc|}
\hline Case \# & $\lambda_{1}$ & $\lambda_{2}$ & $\lambda_{3}$ & $p(1)$ & $p(2)$ & $p(3)$ \\
\hline 0 & 0 & 0 & 0 & $1 / 3$ & $1 / 3$ & $1 / 3$ \\
1 & $\infty$ & $\infty$ & $\infty$ & 0.5 & 0.5 & 0 \\
2 & 0.4 & $\infty$ & $\infty$ & 0.41 & 0.59 & 0 \\
3 & 0.4 & 0.4 & $\infty$ & 0.44 & 0.44 & 0.12 \\
4 & 0 & 0.4 & $\infty$ & $1 / 3$ & 0.44 & 0.23 \\
5 & 0 & 0 & $\infty$ & $1 / 3$ & $1 / 3$ & $1 / 3$ \\
6 & 0.4 & 0.4 & 0.4 & 0.44 & 0.44 & 0.12 \\
\hline
\end{tabular}

Observe that each option is the best only in one of the three states, so under full information (Case 0 ), $p(i)=\frac{1}{3}$ for $i=1,2,3$. In contrast, if the consumer does not process any information at all (Case 1), then she would choose either Product 1 or Product 2, but never Product 3 since its expected value is lower that the other two (actually she is indifferent to PRODUCT 1 and 2, and here we assume she chooses each one with equal probability). At a first glance, it might seem intuitively appealing that reducing the information cost of either PRODUCT 1 or PRODUCT 2 should increase its choice probability, since the consumer would be able to more confidently assess it as the better of the two options. However, this is not correct, as seen in Case 2. When $\lambda_{1}=0.4, \lambda_{2}=\lambda_{3}=\infty$, the consumer chooses PRODUCT 1 less $(p(1)<p(2))$. This is because with reduced information cost, the consumer is also able to learn with more confidence the states in which PRODUCT 1 is not the best option. Note that Product 3 is still not chosen since in expectation it is inferior to Product 2. 
However, this changes when the information cost of PRODUCT 2 is also reduced, as in Case 3 with $\lambda_{1}=\lambda_{2}=0.4$ and $\lambda_{3}=\infty$. Now, the consumer selects PRODUCT 1 and 2 with equal probability. More interestingly, even though she does not process any information about ProduCT 3 directly, from the inferential information obtained through Product 1 and Product 2, she is able to identify cases where it is likely to be the best alternative. As a result, $p(3)$ increases to 0.125 .

Let us now explore the impact of freely available information. As seen in Case 4, when the consumer knows the exact value of PRODUCT 1 , she chooses it only when $v_{1}=1$ (hence $p(1)$ further reduces to $1 / 3$ ). On the other hand, conditional on $v_{1}=0$, the expected value of ProduCT 2 is higher than that of Product 3 and given that she processes information on Product 2, she chooses it with higher probability. Interestingly, the fact that she has perfect information about Product 1 mainly benefits Product 3. This is because in this example there are three products and only three states. Hence, it is sufficient for the DM to evaluate Product 1 and Product 2 independently (form opinions about each taking a non-zero value). Given that there are only 3 states possible, she need not process additional information to learn about ProduCT 3. Consequently, knowing more confidently that she is facing states 2 or 3 does not significantly alter how she will process information about Product 2 ( $\lambda_{2}$ is the same in Cases 3 and 4$)$ - she still has to evaluate PRODUCT 2 and form opinion about its value being 1 or 0 . Of course, if the cost of information for PRoduct 2 reduces, she will be able to make this assessment better and the conditional choice probability for PRODUCT 2 would decrease, as confirmed by Case 5 . The fact that the consumer does not need to process information about PRODUCT 3 beyond what she learns and infers through Product 1 and 2 is substantiated by Case 6. Comparing it with Case 3 confirms that $\lambda_{3}$ has no impact on the choice probability for this example (as long as it is equal or higher than $\lambda_{2}$ ).

Duplicate Alternatives. It has been shown that when the information costs are uniform, the resulting choice behavior of rationally inattentive consumers (i.e. GMNL) does not suffer from the IIA property. Specifically, Matějka and McKay (2015) establish that duplicate alternatives are jointly treated as one alternative. Two alternatives are referred to as "duplicates" if in the prior beliefs of the customers, the probability of the two alternatives taking different values is zero in all states of the world. Recognizing that information costs of duplicate options can indeed be different, we now explore the extension of this result to the case of differentiated information costs.

To this end, we investigate what happens when a duplicate alternative is added to the choice set. Suppose that a new product, say PRODUCT $\hat{2}$, is added to the existing set of products $\mathcal{A}=$ $\{1,2,3\}$. Let $\hat{\mathcal{A}}$ denote the choice set which includes Product $\hat{2}$, i.e., $\hat{\mathcal{A}}=\mathcal{A} \cup\{\hat{2}\}$. Moreover, define $\hat{p}(\mathbf{u}), \mathbf{u} \in \mathbb{R}^{4}$, as the prior belief of the inattentive consumer choosing a product in $\hat{A}$. The prior $\hat{p}(\mathbf{u})$ is obtained from $p(\mathbf{v}), \mathbf{v} \in \mathbb{R}^{3}$, by simply duplicating Product $2 \in \mathcal{A}$, i.e., $p(\mathbf{v})$ is the marginal 
distribution of $\hat{p}(\mathbf{u})$ with respect to $u_{\hat{2}}$. Note that since the values of the duplicate products in $\hat{\mathcal{A}}$ are fully correlated, the consumer will only process information about the cheaper cost duplicate and this would yield the exact same information about the other duplicate. Hence, even if the individual information costs might differ, the consumer remains indifferent between PRODUCT 2 and Product $\hat{2}$ in $\hat{\mathcal{A}}$. Consequently, the probability of choosing Product 2 or Product $\hat{2}$ among available alternatives in $\hat{\mathcal{A}}$ exactly equals the choice probability for PRODUCT 2 among alternatives in $\mathcal{A}$, provided that it is not cheaper for the consumer to process information about PRODUCT $\hat{2}$ than about PRODUCT 2, i.e., if $\lambda_{\hat{2}} \geq \lambda_{2}$. Proposition 3 formally presents this result for the general case with $n$ alternatives.

Proposition 3. Let $\{p(i \mid \mathbf{v})\}_{i \in \mathcal{A}}$ for all $\mathbf{v} \in \mathbb{R}^{n}$ be the set of optimal conditional choice probabilities for an inattentive consumer choosing from available alternatives in $\mathcal{A}$. If a new alternative $\hat{\jmath}$, which is the duplicate of an existing alternative $\jmath \in \mathcal{A}$, with information cost $\lambda_{\hat{\jmath}} \geq \lambda_{\jmath}$ is also offered to the consumer, then the set of optimal conditional choice probabilities, $\{\hat{p}(i \mid \mathbf{u})\}_{i \in \mathcal{A} \cup \hat{\jmath}}$ for all $\mathbf{u} \in \mathbb{R}^{n+1}$, satisfies

$$
\hat{p}(i \mid \mathbf{u})=p(i \mid \mathbf{v}) \quad \text { for all } i \notin\{\jmath, \hat{\jmath}\}
$$

and

$$
\hat{p}(\jmath \mid \mathbf{u})+\hat{p}(\hat{\jmath} \mid \mathbf{u})=p(\jmath \mid \mathbf{v}),
$$

whenever $v_{i}=u_{i}$ for all $i \notin\{\jmath, \hat{\jmath}\}$.

We should mention that analogous equalities also hold for the unconditional choice probabilities in the above proposition. Further, the requirement that $\lambda_{\hat{\jmath}} \geq \lambda_{\jmath}$ is critical - if processing information about the duplicate is cheaper than about the original alternative, the optimal choice may differ since more information is likely to be processed due to the availability of a cheaper information source. The above also implies that when duplicates exist in the choice set, it is possible to drop the ones with higher information costs from the set and determine the choice probability for the cheapest cost alternative of the duplicates. This choice probability can then be allocated over all duplicate alternatives arbitrarily.

Dominated Alternatives. A closely related notion to duplicates is dominated alternatives. A dominated alternative is one whose value is lower than another alternative in all states of the world. Such alternatives are never selected when the cost of information is uniform across alternatives (Matějka and McKay 2015). It can be easily verified that this extends to the more general case of differentiated information costs (shifting the choice probability to the dominating alternative would increase the consumer's objective function). This does not mean information is not processed about a dominated alternative. As a matter of fact, whether a dominated option is available or not in the 
choice set can become relevant in the case of non-uniform information costs since it might serve as a cheap channel to learn about other options, as is illustrated by the following example.

Example 2 (Strong Failure of Regularity) Consider a consumer visiting a retail store to buy a certain product (e.g. a smart phone) and choosing between either Product 1 or Product 2. She is well-informed about PRODUCT 1 based on past experience, which leads to a safe (deterministic) valuation of 1.0 as given in Table 2. On the other hand, PRODUCT 2 might be better or worse than Product 1 such that there are two equally likely states (State 1 and State 2) corresponding to a higher and a lower value for Product 2 (either 1.2 or 0.6). Suppose that exploring Product 2 in the store is difficult (e.g. lack of sample product on display). Accordingly, let the information cost be $\lambda_{2}=1$. At this cost, she will not acquire information about Product 2 and simply buy ProduCT 1 (refer to Table 2). Now suppose that the store adds Product 3 to its assortment. Although Product 3 has most of the key features of Product 2 (e.g. operating system), it is inferior in some other elements (e.g. build quality, camera resolution). Overall, Product 2 dominates Product 3 in both State 1 and State 2. Assume that a sample for ProDuCT 3 is readily available in the store for customers to inspect, hence the consumer can learn about it at cost $\lambda_{3}=0.2$. Subsequently, the consumer also processes information about PRODUCT 2 by mainly inferring through PRODUCT 3. Although the consumer never chooses the dominated Product 3, the unconditional purchase probability of PRODUCT 2 increases from 0 to 0.29 .

Table 2 Values and unconditional choice probabilities when an inferior product is added

\begin{tabular}{|c|cc||ccc|}
\hline \multirow{2}{*}{ Product } & \multicolumn{2}{|c|}{ Value in } & \multicolumn{2}{c|}{ Unconditional Choice Probabilities when } \\
& State 1 & State 2 & $\mathcal{A}=\{1,2\}$ & $\mathcal{A}=\{1,2,3\}$ \\
\hline 1 & 1 & 1 & 1.00 & 0.71 \\
2 & 0.6 & 1.2 & 0.00 & 0.29 \\
3 & 0.5 & 0.9 & - & 0.00 \\
\hline
\end{tabular}

The above example highlights failure of the regularity condition put forth by Luce and Suppes (1965), which requires that adding a product to the choice set does not increase the market share of another product. Matějka and McKay (2015) show that a rationally inattentive consumer facing uniform information costs might fail the regularity condition. This is because introducing a new product can set incentives for the consumer to get information about the new product in a way that she is also informed about a previously "uninteresting" product. With this additional information, she might identify cases where she buys the previously uninteresting product. If the new product is inferior (i.e., dominated), however, the consumer would completely disregard the new product and also would not process any information about it. Hence, there is no failure of regularity under 
Huettner, Boyacı, and Akçay: Consumer Choice When Options Have Different Information Costs

uniform information costs when the added product is dominated. In contrast, we show that nonuniform information costs can induce failure of regularity even if the inclusion is an inferior, neverselected alternative (hence our usage of the term strong failure of regularity).

There is a long standing discussion about the concept of failure of regularity. One explanation is the asymmetric dominance effect (Huber et al. 1982), which requires that the inferior product is only dominated by one of the alternative products. Note that this is not the case in our example; Product 3 is dominated by both Product 2 and Product 1. Another popular explanation is extremeness aversion (Simonson and Tversky 1992), where it is assumed that the consumer buys from the middle. Also menu/halo effects, where the addition of a product signals the quality of the producer, can induce strong failure of regularity (Luce and Raiffa 1957). Such explanations suggest that the consumer's preferences somehow "change" by the introduction of further alternatives. In stark contrast, in our model, the consumer updates her belief about the value of the alternatives in a way that is completely consistent with her prior. ${ }^{6}$ Rather, it is the rational inattentiveness of the customer, coupled with asymmetric information costs that leads to the strong failure of regularity. To the best of our knowledge, this is a new explanation for this well-documented phenomenon.

\section{General Choice Behavior: ReD-Bus/Blue-Bus}

We now demonstrate the choice behavior of rationally inattentive customers under a general setting with different degrees of correlations among the products and non-uniform information costs. For this purpose we consider the classic red-bus/blue-bus problem, and adopt the primary setup in Matějka and McKay (2015). The inattentive consumer, whom we refer to as the decision-maker $(\mathrm{DM})$ in this particular context, faces three alternatives - she may take the TRAIN $(T)$, the BLUE BUS $(B)$, or the RED BUS $(R)$. Table 3 gives the four possible states of nature $\mathbf{v}=\left(v_{T}, v_{R}, v_{B}\right)$, and prior belief of the decision-maker about each state, where $\rho$ denotes the correlation between the values of the two buses (values of 0 and 1 indicate that the particular bus is "slow" or "fast", respectively).

\section{Table 3 Possible states and prior beliefs}

\begin{tabular}{|c|cccc|}
\cline { 2 - 5 } \multicolumn{1}{c|}{} & State 1 & State 2 & State 3 & State 4 \\
\hline TRAIN & $\frac{1}{2}$ & $\frac{1}{2}$ & $\frac{1}{2}$ & $\frac{1}{2}$ \\
RED BUS & 0 & 1 & 0 & 1 \\
BLUE BUS & 0 & 0 & 1 & 1 \\
\hline$p(\mathbf{v})$ & $\frac{1}{4}(1+\rho)$ & $\frac{1}{4}(1-\rho)$ & $\frac{1}{4}(1-\rho)$ & $\frac{1}{4}(1+\rho)$ \\
\hline
\end{tabular}

\footnotetext{
${ }^{6}$ Even if the information cost of Product 3 approaches zero, Product 2 is not chosen more than $50 \%$ of the time.
} 
Note that the speed of the TRAIN is deterministic $\left(v_{T}=\frac{1}{2}\right)$, and the expected values of all three alternatives are equal to $\frac{1}{2}$. If the decision-maker were to choose an alternative without processing any information, she would be indifferent between the three options, i.e., $p(T)=p(R)=p(B)=\frac{1}{3}$. On the other hand, if the decision-maker could process information freely, she would always choose the fastest option, i.e., $p(T)=\frac{1}{4}(1+\rho)$ and $p(R)=p(B)=\frac{1}{8}(3-\rho)$ (see Figure 2a). Clearly, since the buses are symmetric, they are chosen with equal probability, and this probability decreases as $\rho$ increases (the DM believes increasingly that the buses are similar in speed).

Figure $2 \mathrm{~b}$ illustrates the subtle changes in the DM's choice behaviour when processing information is costly but the costs are symmetric (uniform), i.e., $\lambda_{R}=\lambda_{B}=0.4$. As expected, the two buses are still always selected with equal probabilities, which are non-increasing in $\rho$. Furthermore, both buses are selected more by the DM, compared to the case with freely available information. In particular, when the DM has sufficiently strong belief that one of the buses must be fast, she does not consider the TRAIN as an option (this happens when $\rho \leq-0.31$ ). Given that information processing is costly, due to her beliefs that the TRAIN is unlikely to be the best option, she instead allocates all her time and attention to understand which bus is faster (since the buses are symmetric, each bus is chosen with $50 \%$ chance).

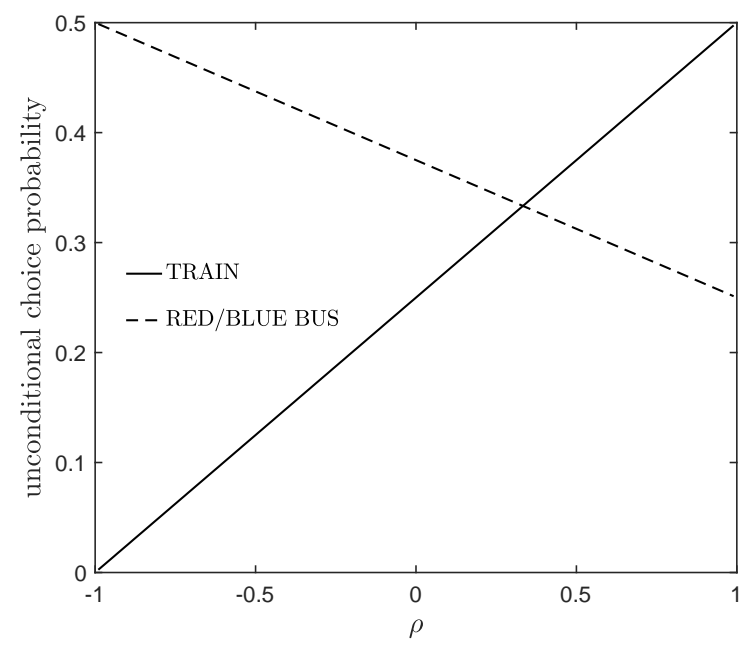

(a) $\lambda_{R}=\lambda_{B}=0$

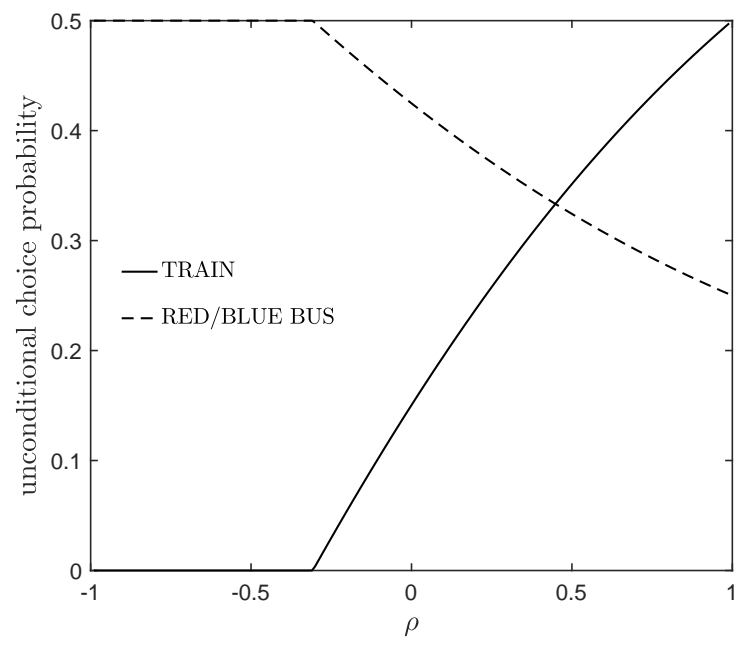

(b) $\lambda_{R}=\lambda_{B}=0.4$

Figure 2 RED BUS/BLUE BUS when information costs are symmetric

Now consider the scenario with asymmetric (non-uniform) information costs. Specifically, suppose that acquiring and processing information about the RED BUS is less expensive than about the BLUE BUS for the DM, i.e., $\lambda_{R} \leq \lambda_{B}$. Based on Proposition 2, the DM's choice is determined by the solution to the following optimization problem

$$
\max _{\left\{p\left(i \mid v_{T}, v_{B}\right)\right\}_{i \in A}} \lambda_{B} \sum_{\mathbf{v}} p(\mathbf{v}) \log \left(\sum_{i \in\{T, B, R\}} e^{\frac{v_{i}}{\lambda_{B}}} p\left(i \mid \frac{1}{2}\right)^{\frac{\lambda_{R}}{\lambda_{B}}} p\left(i \mid\left(\frac{1}{2}, v_{T}\right)\right)^{\frac{\lambda_{B}-\lambda_{R}}{\lambda_{B}}}\right) \quad \text { s.t. }
$$




$$
\begin{aligned}
p\left(i \mid v_{T}, v_{B}\right) \geq 0 & \text { for } i \in\{T, B, R\} \text { and }\left(v_{T}, v_{B}\right) \in\left\{\left(\frac{1}{2}, 0\right),\left(\frac{1}{2}, 1\right)\right\} \\
\sum_{i \in A} p\left(i \mid v_{T}, v_{B}\right) & =1 \quad
\end{aligned}
$$

which can be solved easily by numerical search.

Figure 3a shows the unconditional choice probabilities when $\lambda_{R}=0.2$ and $\lambda_{B}=0.4$. We observe, as in the case of uniform information costs, that the TRAIN is never selected if the DM has sufficient belief that the two bus speeds are negatively correlated (i.e., if one of the buses must be fast). Note that this occurs for a smaller range of beliefs/correlations compared to the case of uniform costs ( $\rho \leq-0.47$ versus $\rho \leq-0.31$ ). Nevertheless, as the DM's prior belief that the two buses are similar gets stronger with $\rho$, she also starts selecting the TRAIN. Interestingly, in this range the DM builds a stronger preference for the "cheap" RED BUS over the BLUE BUS. This is because she acquires more information about the RED BUS and has more confidence about its speed compared to the BLUE BUS. In particular, as $\rho$ approaches 1, the DM believes that the buses have identical speed. Consequently, whenever she decides to take a bus, she takes the RED BUS, on which she has more information. This signifies the importance of information provision for a seller in forming its product choice set. When the alternatives are very similar in the eyes of the DM, even a slight improvement in the provision of information for one product can significantly shift demand towards it. This is particularly stark considering that when $\rho=1$, the DM treats duplicate options jointly as one.

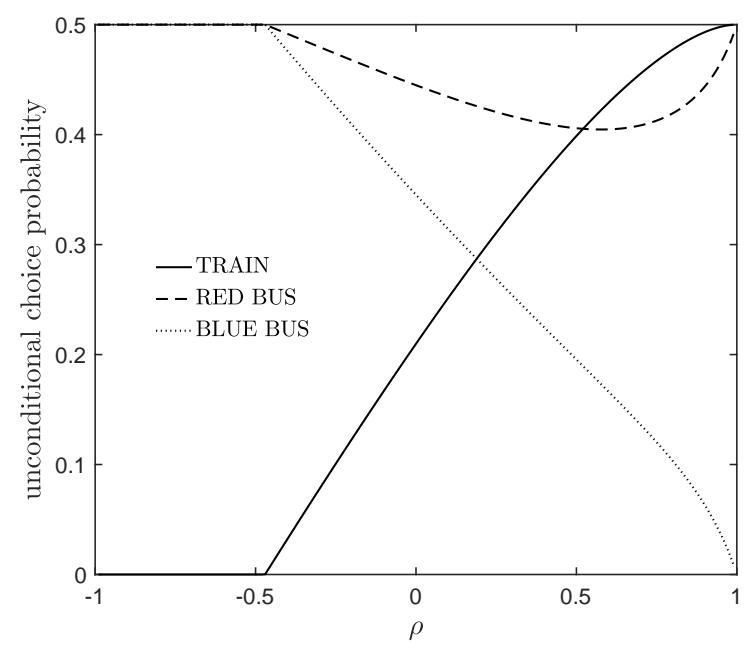

(a)

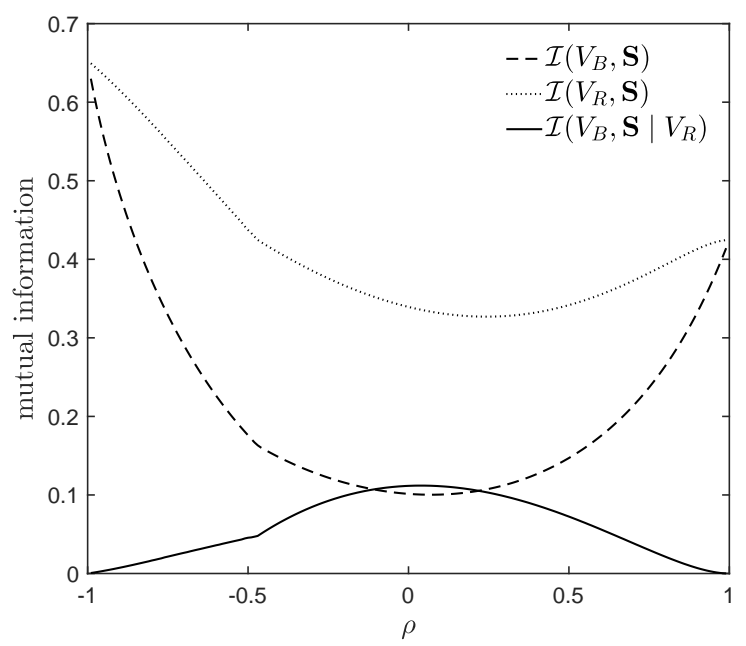

(b)

Figure 3 RED BUS/BLUE BUS when information costs are asymmetric $\left(\lambda_{R}=0.2\right.$ and $\left.\lambda_{B}=0.4\right)$

We can delineate the driving forces of this behavior by directly investigating the attention allocation and information acquisition strategies of the DM. For this purpose, Figure 3b depicts the mutual information and the conditional mutual information that is processed by the DM. Naturally, 
since the information is more readily available about the RED BUS, the DM learns more about it; i.e., $\mathcal{I}\left(V_{R}, \mathbf{S}\right)>\mathcal{I}\left(V_{B}, \mathbf{S}\right)$. Furthermore, part of what the DM learns about the BLUE Bus is inferential information, while the rest is direct information. The latter, which quantifies the amount of effort the DM spends to learn about the BLUE BUS beyond what she can infer from studying the RED BUS, is the conditional mutual information $\mathcal{I}\left(V_{B}, \mathbf{S} \mid V_{R}\right)$. Observe that $\mathcal{I}\left(V_{B}, \mathbf{S} \mid V_{R}\right)$ approaches zero as $\rho \downarrow-1$ and $\rho \uparrow 1$. This is because, when $\rho \downarrow-1$, the DM closely studies the RED BUS and if she concludes that it must be fast, she simply takes it, whereas if she concludes that it must be slow, she automatically takes the BLUE BUS (since she simply infers and then strongly believes that it must be fast). On the other extreme, when $\rho \uparrow 1$, the DM closely studies the RED BUs and if she concludes that it is most probably fast, she simply takes it, whereas if she concludes that it must be slow, she automatically takes the TRAIN (since she infers that the BLUE BUS must be slow too). In contrast, when $\rho$ is around zero, the decision-maker cannot infer sufficient information about the BLUE BUS through the information processed about the RED BUS, and decides to pay additional attention and acquire direct information about the BLUE BUS to make the optimal choice. ${ }^{7}$

In order to deepen our understanding of the DM's choice behaviour when information costs are asymmetric, we next focus on the conditional choice probabilities given in Figures 4 and 5. As seen in Figure 4a, even when the TRAIN is the best option, it is not selected by the DM for sufficiently negative $\rho, \rho \leq-0.47$ (as previously explained). Moreover, in this range, her conditional belief for the RED BUS being slow yet the BLUE BUs being fast is decreasing in $\rho$. Therefore, the conditional choice probability of RED BUS (resp. BLUE BUS) increases (resp. decreases) in $\rho$. On the other hand, for $\rho>-0.47$, the TRAIN is also chosen and the decision-maker increasingly prefers the TRAIN and avoids the buses as $\rho$ increases. Further she also learns that both buses are more likely to be slow mainly by processing direct information about the (cheaper) RED BUS. Hence, as long as $\rho \leq 0.60$, the DM takes the RED BUS less often than the BLUE BUS. Interestingly, for high levels of $\rho$, when the DM erroneously believes that the RED BUS is fast, she also infers that the BLUE BUS must also be fast but since she is more informed about the RED BUS, she takes it. In this case, the BLUE BUS is rarely chosen. In contrast, when both buses are fast, i.e., $v_{R}=v_{B}=1$, as in Figure $4 \mathrm{~b}$, the DM always prefers the RED BUS over the BLUE BUS due to its information advantage. In this case, she rarely makes the wrong decision by taking the TRAIN. Further, only if the correlation $\rho$ is around zero, the DM processes direct information about both buses and may follow a positive signal concerning the BLUE BUS when receiving an erroneous negative signal about the RED BUS. The selection probability of the BLUE BUS is highest in this range, i.e., the information cost disadvantage for a fast BLUE BUS is low if little can be inferred about it from knowing the RED BUS.

${ }^{7}$ Concerning the fact that $\mathcal{I}\left(V_{B}, \mathbf{S} \mid V_{R}\right)>\mathcal{I}\left(V_{B}, \mathbf{S}\right)$ for $\rho \approx 0$, we refer to the discussion of Equation (6). 


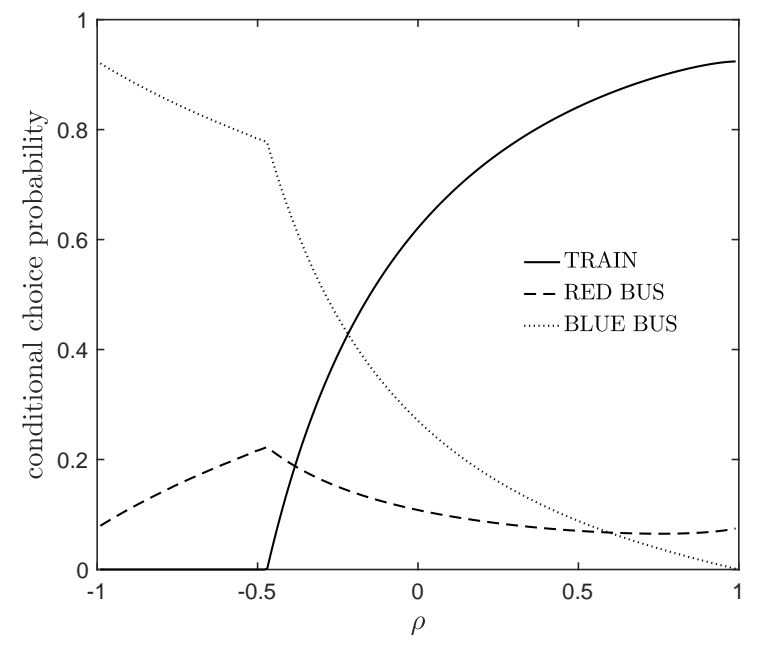

(a) $\left(v_{T}, v_{R}, v_{B}\right)=\left(\frac{1}{2}, 0,0\right)$

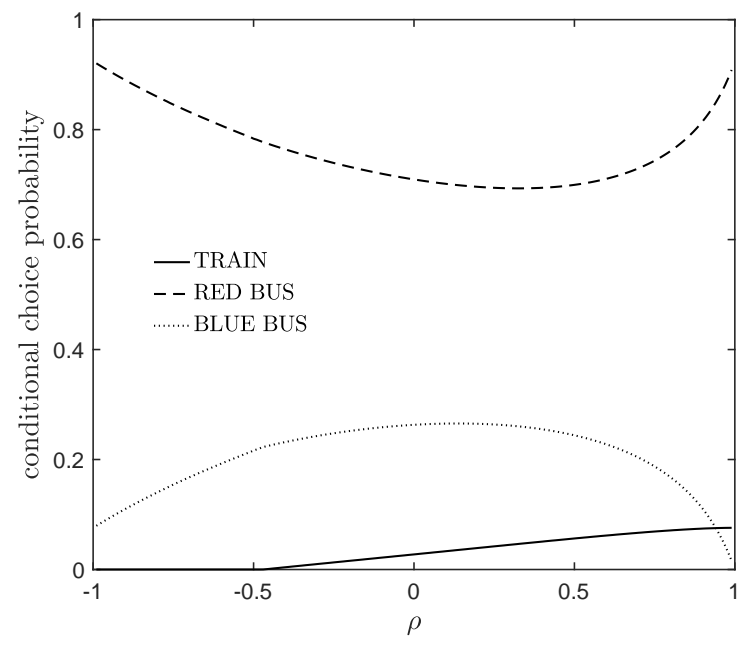

(b) $\left(v_{T}, v_{R}, v_{B}\right)=\left(\frac{1}{2}, 1,1\right)$

Figure 4 Conditional choice probabilities for RED BUS/BLUE BUS when the two buses are equally fast

Figure 5a confirms that the DM most often makes the right choice when information processing is cheap for the fast bus (RED BUS) and expensive for the slow bus (BLUE BUS), i.e., $v_{R}=1$ and $v_{B}=0$. As shown in Figure $5 \mathrm{~b}$, this remains the case even if it is harder to acquire information about the fast bus $v_{R}=0$ and $v_{B}=1$, provided that the DM has negatively correlated beliefs. When the DM increasingly believes the buses to be similar ( $\rho$ increases), however, the likelihood of taking the BLUE BUS decreases sharply. This is because the DM has more information about the slow RED BUS, and since the buses are very similar according to her beliefs, she draws the inference that the BLUE BUS must also be slow. She instead increasingly chooses the TRAIN (and makes the wrong decision). In the extreme case, $\rho \geq 0.95$ the likelihood of her taking the correct BLUE BUS is even less than the RED BUS.

From the above discussion, it is clear that reducing the information cost of an alternative results in more information to be processed by the DM. This means that the net-payoff to the DM also improves after optimal processing of information. However, it is also evident that this does not mean that the DM makes more correct choices all the time. To highlight this fact, we depict in Figure 6, the conditional probability of taking "a bus" (red or blue). We know that when the decision maker faces uniform information costs $\left(\lambda_{R}=\lambda_{B}=0.4\right)$, she takes the bus too often (see also Figure 2 and the related discussion). One could conjecture that reducing the information cost of even one alternative increases the amount of information processed, so it should bring this probability close to the perfect information case $\left(\lambda_{R}=\lambda_{B}=0\right)$. From Figure 6 , it is evident that this is only partially correct. For negative correlation levels, the DM more correctly identifies the TRAIN as the correct option. However, at positive correlation levels a new decision bias is created. Since the DM knows 


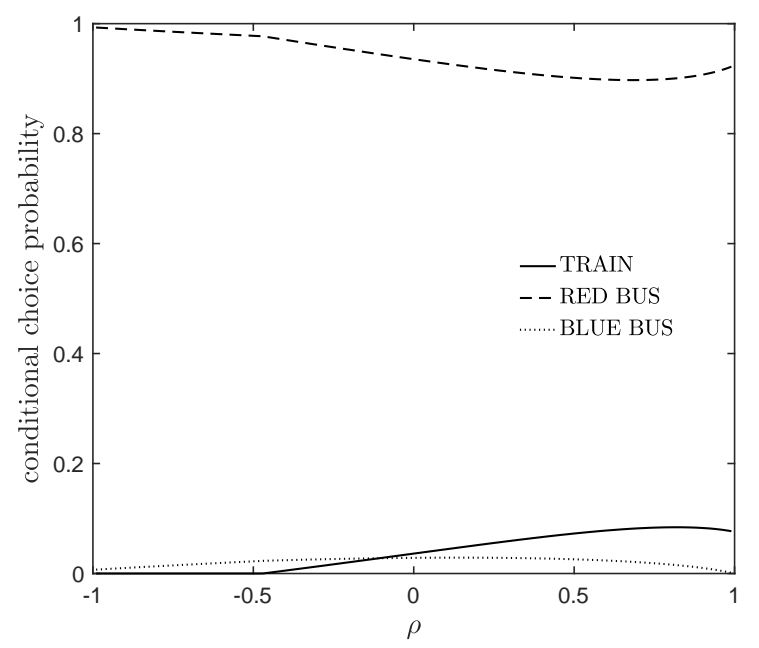

(a) $\left(v_{T}, v_{R}, v_{B}\right)=\left(\frac{1}{2}, 1,0\right)$

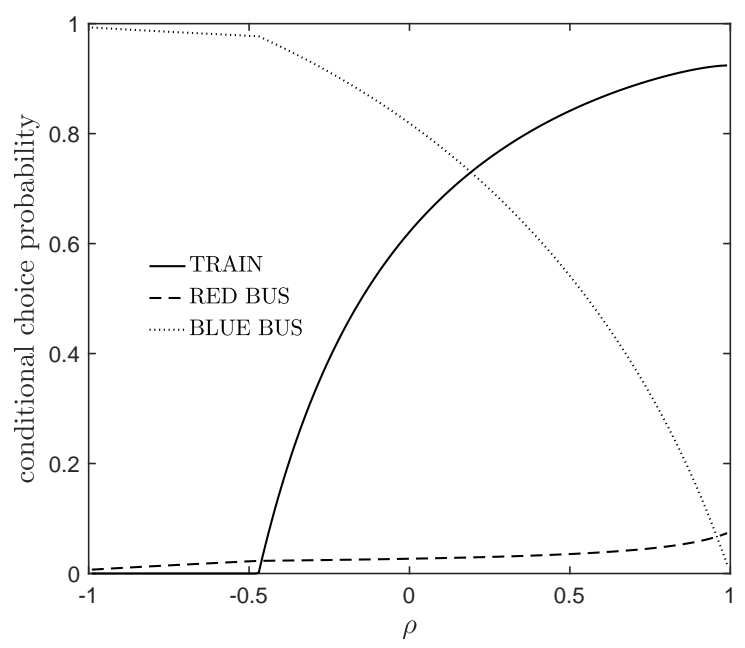

(b) $\left(v_{T}, v_{R}, v_{B}\right)=\left(\frac{1}{2}, 0,1\right)$

Figure 5 Conditional choice probabilities for RED BUS/BLUE BUS when the two buses are different

more about the bus with the lower cost, she starts drawing strong (and wrong) inferences about the other bus, and this time she ends up taking the TRAIN too often. Reducing the information cost of the BLUE BUS so that information costs are uniform again $\left(\lambda_{R}=\lambda_{B}=0.2\right)$ eliminates this decision bias and brings the conditional choice probability of choosing either bus closer to the perfect information case for all levels of $\rho$. This highlights the benefits that a seller can potentially earn from presenting information about different choice options in a rather similar and uniform manner.

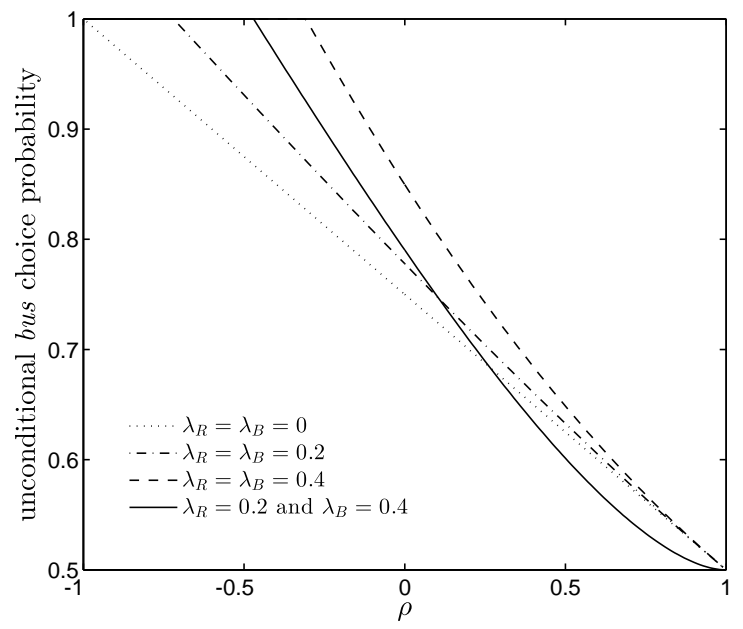

Figure 6 Unconditional choice probabilities for choosing either the RED BUS or the BLUE BUS

\section{Concluding Remarks}

In this paper, we develop a consumer choice model where rationally inattentive customers choose among a given set of alternatives. Our novel contribution is the incorporation of information costs 
that differ among the alternatives. This captures the notion that it might be inherently (or by seller design) more difficult to learn about some alternatives than about others. We develop an information cost function that distinguishes between direct and inferential information obtained by the consumer from studying each alternative, and that prioritizes the use of cheaper sources in the acquisition and processing of information. This conditional mutual information based function generalizes the Shannon cost functions commonly utilized in the rational inattention literature. We analyze the choice problem of the consumer and show that the optimal choice behavior can be characterized analytically. When the unit cost of acquiring information is the same across all alternatives, the choice behavior reduces to the GMNL choice studied by Matějka and McKay (2015). According to the optimal choice behavior, the conditional choice probability associated with each option depends on realized values of the alternatives, their information costs, and prior beliefs. Although the exact relationship is non-trivial, essentially the relative "attractiveness" of each option is adjusted by the fact that the consumer learns more about the alternatives with lower information costs. Accordingly, if the information obtained by these alternatives imply a higher (or lower) likelihood of selecting a particular alternative, it is weighed into the attractiveness of that alternative appropriately.

We study a number of limiting scenarios and typical examples to illustrate the optimal choice behavior, and show that non-uniform information costs can induce complex consumer behaviour. Accordingly, the consequences for the seller depend on the particular situation. Although an asymmetric reduction of information costs yields an overall better-informed consumer, the consumer's beliefs can become strongly biased by focusing on a particular information channel. Perhaps surprisingly, there are situations where the market share of a product may increase when it becomes harder to learn about it. Our characterization enables us to verify if such changes (perhaps due to alterations in the information provision strategy of the seller) would lead to more correct (or incorrect) choices for the consumer, and can be used to evaluate the benefits (or losses) to the seller. We also identify that if two products are very similar in nature, a difference in information costs typically leads to a striking change in relative market share. This is because the consumer then mainly relies on information about the product with low information cost and forms her belief about the product with high information cost based on inferential information. As both products are similar in quality, she prefers the product of which she is more confident about. In addition to above, our model provides an explanation for strong failure of regularity in consumer behavior, a well-documented phenomenon that occurs if the addition of an inferior - dominated, never selected - product increases the market share of another product. This may occur if the newly introduced inferior product facilitates an easier access to information about existing products. To the best of our knowledge, this explanation for the strong failure of regularity has not been discussed in the extant literature. 
Most of the decisions that consumers have to make require time, attention and cognitive effort, all of which are limited resources. Our model offers a micro-founded description of how such choices are made when the consumers trade off the value of better information against the costs, in a context where information can be acquired about the alternatives with different rates of time-and-attentionefficiency. As noted earlier, this choice behavior and the resulting description of demand is a crucial input to many practical operational problems. As a concluding example, consider an online firm like airbnb.com or booking.com. When consumers search for a particular accommodation, there are usually a large number of potential hits. It is well-known that people do not have the time and attention span to go through all pages. What is often displayed on the first page (or even a subset of this page) is where most attention is directed, while choices listed on the following pages require additional effort to evaluate. From the seller's revenue management perspective, it is extremely important to decide on the order at which options are displayed. Determining this requires a consumer demand model that describes how choices are going to be made when the cost of information differs among the options and the consumer is rational and efficient when evaluating her options. Going a step of further, such sellers face the trade-off between displaying more options on the same page with less related information (high information costs) versus less options but with more available information (low information costs). Our choice model has the potential to serve as the building block of such product assortment, ordering and strategic information provisioning decisions.

It is worth pointing out that real-life practical applications of our consumer choice model would benefit from two key developments. The first one pertains to the empirical validation of rational inattention and estimation of the choice model. Fortunately, there is a fast growing recent interest in the economics literature on rational inattention and breakthroughs are being made in both theoretical and empirical directions. In recent work, Caplin and Dean (2015) describe a method to identify whether a decision maker is rational inattentive or not from state dependent choice data. In a similar vein, Oliveira et al. (2016) introduce a method to elicit preferences and to estimate the information cost function of a rationally inattentive decision maker. Their setup works for what they call canonical information cost functions, to which our generalized Shannon information cost function belongs. The second development required pertains to solution methods. It is well-known that assortment optimization problems, even under simple variations of the multinomial logit choice, are very difficult to solve (they are often NP-hard or NP-complete). The generalized GMNL choice model we develop adds additional computational challenges. Therefore, in order to solve realisticallysized practical problems, it is necessary to develop an efficient algorithm to first solve the consumer choice model and then embed it in pricing and/or assortment optimization. This is subject of our ongoing research. 
Huettner, Boyacı, and Akçay: Consumer Choice When Options Have Different Information Costs

Article submitted to ; manuscript no. (Please, provide the manuscript number!)

\section{References}

Alptekinoğlu, A., J. H. Semple. 2015. The exponomial choice model: A new alternative for assortment and price optimization. Operations Research 64(1) 79-93.

Anderson, S. P., A. de Palma, J-F. Thisse. 1992. Discrete Choice Theory of Product Differentiation. MIT Press, Cambridge.

Blanchet, J. H., G. Gallego, V. Goyal. 2016. A markov chain approximation to choice modeling. Operations Research 64(4) 886-905.

Boyacı, T., Y. Akçay. 2016. Pricing when customers have limited attention. Working paper, ESMT Berlin.

Branco, F., M. Sun, J. M. Villas-Boas. 2012. Optimal search for product information. Management Science 58(11) 2037-2056.

Caplin, A., M. Dean. 2015. Revealed preference, rational inattention, and costly information acquisition. American Economic Review 105(7) 2183-2203.

Cover, T. M., J. A. Thomas. 2006. Elements of Information Theory. 2nd ed. Wiley, New York.

Csiszár, I. 2008. Axiomatic characterizations of information measures. Entropy 10(3) 261-273.

Davis, A. M., E. Katok, A. M. Kwasnica. 2014. Should sellers prefer auctions? a laboratory comparison of auctions and sequential mechanisms. Management Science 60(4) 990-1008.

Dong, L., P. Kouvelis, Z. Tian. 2009. Dynamic pricing and inventory control of substitute products. Manufacturing $\&$ Service Operations Management 11(2) 317-339.

Gabaix, X. 2014. A sparsity-based model of bounded rationality. The Quarterly Journal of Economics 129(4) 1661-1710.

Hamilton, R. W., D. V. Thompson. 2007. Is there a substitute for direct experience? Comparing consumers' preferences after direct and indirect product experiences. Journal of Consumer Research 34(4) 546-555.

Hanson, W., K. Martin. 1996. Optimizing multinomial logit profit functions. Management Science 42(7) 992-1003.

Huber, J., J. W. Payne, C. Puto. 1982. Adding asymmetrically dominated alternatives: Violations of regularity and the similarity hypothesis. Journal of Consumer Research 9(1) 90-98.

Ke, T. T., Z-J M. Shen, J. M. Villas-Boas. 2016. Search for information on multiple products. Management Science Forthcoming.

Luce, R. D., H. Raiffa. 1957. Games and Decisions. Wiley, New York.

Luce, R. D., P. Suppes. 1965. Preference, utility, and subjective probability, Handbook of Mathematical Psychology, vol. 3. Wiley, New York.

Maćkowiak, B., M. Wiederholt. 2009. Optimal sticky prices under rational inattention. American Economic Review 99(3) 769-803. 
Manzini, P., M. Mariotti. 2014. Stochastic choice and consideration sets. Econometrica 82(3) 1153-1176.

Matějka, F. 2015. Rigid pricing and rationally inattentive consumer. Journal of Economic Theory 158 656-678.

Matějka, F., A. McKay. 2012. Simple market equilibria with rationally inattentive consumers. American Economic Review 102(3) 24-29.

Matějka, F., A. McKay. 2015. Rational inattention to discrete choices: A new foundation for the multinomial logit model. American Economic Review 105(1) 272-298.

McGill, W. J. 1954. Multivariate information transmission. Psychometrika 19 97-116.

Oliveira, H., T. Denti, M. Mihm, K. Ozbek. 2016. Rationally inattentive preferences and hidden information costs. Theoretical Economics forthcoming.

Reis, R. 2006. Inattentive producers. The Review of Economic Studies 73(3) 793-821.

S. J. Hoch, Y-W. Ha. 1986. Consumer learning: Advertising and the ambiguity of product experience. Journal of Consumer Research 13(2) 221-233.

Shannon, C. E. 1948. A mathematical theory of communication. The Bell System Technical Journal 27(3) $379-423$.

Simon, H. A. 1955. A behavioral model of rational choice. The Quarterly Journal of Economics 69(1) $99-118$.

Simon, H. A. 1979. Information processing models of cognition. Annual review of psychology 30(1) 363-396.

Simonson, I., A. Tversky. 1992. Choice in context: Tradeoff contrast and extremeness aversion. Journal of Marketing Research 29(3) 281-295.

Sims, C. A. 1998. Stickiness. Carnegie-Rochester Conference Series on Public Policy 49(1) 317-356.

Sims, C. A. 2003. Implications of rational inattention. Journal of Monetary Economics 50(3) 665-690.

Sims, C. A. 2006. Rational inattention: Beyond the linear-quadratic case. The American Economic Review 96(2) 158-163.

Srikanth, J., P. Rusmevichientong. 2016. A nonparametric joint assortment and price choice model. Management Science Forthcoming.

Talluri, K., G. van Ryzin. 2004. Revenue management under a general discrete choice model of consumer behavior. Management Science 50(1) 15-33.

Todd, P. M., G. Gigerenzer. 2000. Précis of simple heuristics that make us smart. Behavioral and brain sciences 23(05) 727-741.

van Ryzin, G., S. Mahajan. 1999. On the relationship between inventory costs and variety benefits in retail assortments. Management Science 45(11) 1496-1509. 
Huettner, Boyacı, and Akçay: Consumer Choice When Options Have Different Information Costs

Verrecchia, R. E. 1982. Information acquisition in a noisy rational expectations economy. Econometrica $1415-1430$.

Weitzman, M. L. 1979. Optimal search for the best alternative. Econometrica 47(3) 641-654.

Wierenga, B. 2008. Handbook of marketing decision models. Springer, New York.

Zhang, D., D. Adelman. 2009. An approximate dynamic programming approach to network revenue management with customer choice. Transportation Science 43(3) 381-394.

\section{Appendix A: Proofs}

\section{Proof of Lemma 1}

The proof is by contradiction. Suppose that $i \in \mathcal{A}$ is $\operatorname{such}$ that $p_{f^{*}}(i, \mathbf{v})>0$, and that there exist $S_{i}^{1}, S_{i}^{2} \subseteq S_{i}$, satisfying $\int_{\mathbf{v} \in \mathbb{R}^{n}} \int_{\mathbf{s} \in S_{i}^{1}} f^{*}(d \mathbf{s}, d \mathbf{v})>0, \int_{\mathbf{v} \in \mathbb{R}^{n}} \int_{\mathbf{s} \in S_{i}^{2}} f^{*}(d \mathbf{s}, d \mathbf{v})>0$, and $f_{\mathbf{V} \mid \mathbf{s}^{1}}^{*} \neq f_{\mathbf{V} \mid \mathbf{s}^{2}}^{*}$ for all $\mathbf{s}^{1} \in S_{i}^{1}, \mathbf{s}^{2} \in S_{i}^{2}$. We can construct a better information strategy $h$ as follows. Pick some $\hat{\mathbf{s}} \in S_{i}^{1} \cup S_{i}^{2}$. Define $h$ by setting for all v:

$$
\begin{aligned}
h(\mathbf{s}, \mathbf{v}): & =f^{*}(\mathbf{s}, \mathbf{v}) \text { for all } \mathbf{s} \notin\left(S_{i}^{1} \cup S_{i}^{2}\right), \\
h_{\mathbf{S} \mid \mathbf{v}}(\hat{\mathbf{s}}): & =\int_{\mathbf{s} \in S_{i}^{1}} f_{\mathbf{S} \mid \mathbf{v}}^{*}(d \mathbf{s})+\int_{\mathbf{s} \in S_{i}^{2}} f_{\mathbf{S} \mid \mathbf{v}}^{*}(d \mathbf{s}), \text { and } \\
h_{\mathbf{S} \mid \mathbf{v}}(\mathbf{s}): & =0 \text { for all } \mathbf{s} \in\left(S_{i}^{1} \cup S_{i}^{2}\right) \backslash\{\hat{\mathbf{s}}\} .
\end{aligned}
$$

Note that $h$ is consistent with $p$. Matějka and McKay (2015) establish that $h$ yields a revenue at least as high as the revenue of $f^{*}$, i.e., $\int_{\mathbf{v} \in \mathbb{R}^{n}} \int_{\mathbf{s} \in \mathbb{R}^{n}} U\left(h_{\mathbf{V} \mid \mathbf{s}}\right) h_{\mathbf{S} \mid \mathbf{v}}(d \mathbf{s}) p(d \mathbf{v}) \geq \int_{\mathbf{v} \in \mathbb{R}^{n}} \int_{\mathbf{s} \in \mathbb{R}^{n}} U\left(f_{\mathbf{V} \mid \mathbf{s}}\right) f_{\mathbf{S} \mid \mathbf{v}}^{*}(d \mathbf{s}) g(d \mathbf{v})$.

We next compare the information costs of $h$ and $f^{*}$. Since the difference between the mutual information of $h$ and $f^{*}$ stems from where the distributions differ, it is helpful to make use of the the probability distributions restricted to this domain. More precisely, we construct a probability distribution $\left.g\right|_{S_{i}^{1} \cup S_{i}^{2}, \mathbf{V}_{1} . . \ell}$ on the restricted domain $S_{i}^{1} \cup S_{i}^{2} \times \mathbf{V}_{1 . . \ell}$ from $g$ by rescaling to $\int_{\mathbf{s} \in S_{i}^{1} \cup S_{i}^{2}, \mathbf{v} \in \mathbf{V}_{1 . \ell}} g(\mathbf{s}, \mathbf{v}) d \mathbf{s} d \mathbf{v}$. Formally, let $\left.f^{*}\right|_{S_{i}^{1} \cup S_{i}^{2}, \mathbf{V}_{1}, . \ell}$ denote the probability distribution obtain from $f^{*}$ as

$$
\left.f^{*}\right|_{S_{i}^{1} \cup S_{i}^{2}, \mathbf{V}_{1 . . \ell}}(\mathbf{s}, \mathbf{v})=\frac{f^{*}(\mathbf{s}, \mathbf{v})}{\int_{\mathbf{s} \in S_{i}^{1} \cup S_{i}^{2}, \mathbf{v} \in \mathbf{V}_{1 . . \ell}} f^{*}(d \mathbf{s}, d \mathbf{v})} \quad \text { for all } \mathbf{s} \in S_{i}^{1} \cup S_{i}^{2}, \mathbf{v} \in \mathbf{V}_{1 . . \ell} .
$$

Analogously, we define $\left.h\right|_{S_{i}^{1} \cup S_{i}^{2}, \mathbf{v}_{1} . . \ell}$.

Dividing the following equation by $\int_{\mathbf{s} \in S_{i}^{1} \cup S_{i}^{2}, \mathbf{v} \in \mathbf{V}_{1} . . \ell} f^{*}(d \mathbf{s}, d \mathbf{v})$,

$$
\begin{aligned}
& \mathcal{I}_{f^{*}}\left(V_{\ell}, \mathbf{S} \mid \mathbf{V}_{1 . . \ell-1}\right)-\mathcal{I}_{h}\left(V_{\ell}, \mathbf{S} \mid \mathbf{V}_{1 . . \ell-1}\right) \\
= & \int_{\mathbf{v}_{1} \ldots \ell, \mathbf{s}} f^{*}\left(\mathbf{s}, \mathbf{v}_{1 . . \ell}\right)\left(\log \frac{f_{\mathbf{S} \mid \mathbf{v}_{1 . \ell \ell}}^{*}(\mathbf{s})}{f_{\mathbf{S} \mid \mathbf{v}_{1} \ldots \ell-1}^{*}(\mathbf{s})}\right) d \mathbf{s} d \mathbf{v}_{1 \ldots \ell}-\int_{\mathbf{v}_{1} \ldots \ell, \mathbf{s}} h_{\mathbf{S} \mid \mathbf{v}_{1} \ldots \ell}(\mathbf{s})\left(\log \frac{h_{\mathbf{S} \mid \mathbf{v}_{1 . \ell \ell}}(\mathbf{s})}{h_{\mathbf{S} \mid \mathbf{v}_{1} \ldots \ell-1}(\mathbf{s})}\right) d \mathbf{s} d \mathbf{v}_{1 \ldots \ell} \\
& \stackrel{(18)-(20)}{=} \int_{\mathbf{v}_{1} \ldots \ell, \mathbf{s} \in S_{i}^{1} \cup S_{i}^{2}} f^{*}\left(\mathbf{s}, \mathbf{v}_{1 . . \ell}\right)\left(\log \frac{f_{\mathbf{S}_{\mid} \mid \mathbf{v}_{1} \ldots \ell}^{*}(\mathbf{s})}{f_{\mathbf{S} \mid \mathbf{v}_{1} \ldots \ell-1}(\mathbf{s})}\right) d \mathbf{s} d \mathbf{v}_{1 \ldots \ell}-\int_{\mathbf{v}_{1} \ldots \ell} h\left(\hat{\mathbf{s}}, \mathbf{v}_{1 \ldots \ell}\right)\left(\log \frac{h_{\mathbf{S} \mid \mathbf{v}_{1} \ldots \ell}(\hat{\mathbf{s}})}{h_{\mathbf{S} \mid \mathbf{v}_{1} \ldots \ell-1}(\hat{\mathbf{s}})}\right) d \mathbf{v}_{1 . . \ell},
\end{aligned}
$$

we get

$$
\begin{aligned}
& X:=\left(\int_{\mathbf{s} \in S_{i}^{1} \cup S_{i}^{2}} f^{*}(d \mathbf{s}, d \mathbf{v})\right)^{-1} \cdot\left[\mathcal{I}_{f^{*}}\left(V_{\ell}, \mathbf{S} \mid \mathbf{V}_{1 \cdot \ell-1}\right)-\mathcal{I}_{h}\left(V_{\ell}, \mathbf{S} \mid \mathbf{V}_{1 \cdot \ell \ell-1}\right)\right]
\end{aligned}
$$

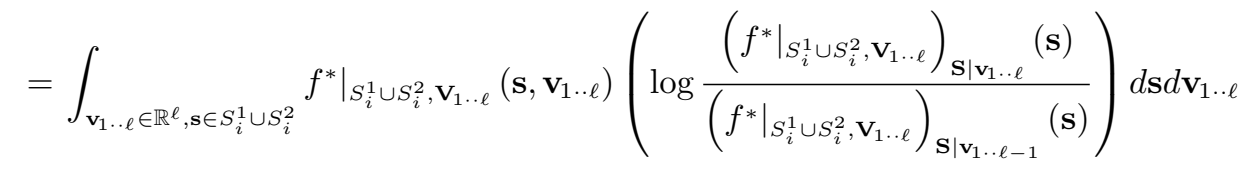




$$
-\left.\int_{\mathbf{v}_{1} . . \ell \in \mathbb{R}^{\ell}} h\right|_{S_{i}^{1} \cup S_{i}^{2}, \mathbf{v}_{1 . . \ell}}\left(\hat{\mathbf{s}}, \mathbf{v}_{1 . . \ell}\right)\left(\log \frac{\left(\left.h\right|_{S_{i}^{1} \cup S_{i}^{2}, \mathbf{v}_{1} . . \ell}\right)_{\mathbf{S} \mid \mathbf{v}_{1 . . \ell}}(\hat{\mathbf{s}})}{\left(\left.h\right|_{S_{i}^{1} \cup S_{i}^{2}, \mathbf{v}_{1} \ldots \ell}\right)_{\mathbf{S} \mid \mathbf{v}_{1} \ldots \ell-1}(\hat{\mathbf{s}})}\right) d \mathbf{v}_{1 . . \ell}
$$

since the ratio of the conditionals within the log terms remains the same when restricting the domain to $S_{i}^{1} \cup S_{i}^{2} \times \mathbf{V}_{1 . . \ell}$ Using $\operatorname{Pr}(x, y)=\operatorname{Pr}(x \mid y) \cdot \operatorname{Pr}(y)$, we get

$$
\begin{aligned}
& X=\left.\int_{\mathbf{v}_{1} \ldots \ell \in \mathbb{R}^{\ell}, \mathbf{s} \in S_{i}^{1} \cup S_{i}^{2}} f^{*}\right|_{S_{i}^{1} \cup S_{i}^{2}, \mathbf{v}_{1 . . \ell}}\left(\mathbf{s}, \mathbf{v}_{1 . . \ell}\right)\left(\log \frac{\left.f^{*}\right|_{S_{i}^{1} \cup S_{i}^{2}, \mathbf{v}_{1 . . \ell}}\left(\mathbf{s}, \mathbf{v}_{1 \ldots \ell}\right)}{\left.f^{*}\right|_{S_{i}^{1} \cup S_{i}^{2}, \mathbf{v}_{1} \ldots \ell}\left(\mathbf{s}, \mathbf{v}_{1 . . \ell-1}\right)}-\log \frac{\left.f^{*}\right|_{S_{i}^{1} \cup S_{i}^{2}, \mathbf{v}_{1 . \ell \ell}}\left(\mathbf{v}_{1 \ldots \ell}\right)}{\left.f^{*}\right|_{S_{i}^{1} \cup S_{i}^{2}, \mathbf{v}_{1} \ldots \ell}\left(\mathbf{v}_{1 \ldots \ell-1}\right)}\right) d \mathbf{s} d \mathbf{v}_{1 . . \ell} \\
& -\left.\int_{\mathbf{v}_{1 . . \ell} \in \mathbb{R}^{\ell}} h\right|_{S_{i}^{1} \cup S_{i}^{2}, \mathbf{v}_{1 . . \ell}}\left(\hat{\mathbf{s}}, \mathbf{v}_{1 . . \ell}\right)\left(\log \frac{\left.h\right|_{S_{i}^{1} \cup S_{i}^{2}, \mathbf{v}_{1 . . \ell}}\left(\hat{\mathbf{s}}, \mathbf{v}_{1} \ldots \ell\right)}{\left.h\right|_{S_{i}^{1} \cup S_{i}^{2}, \mathbf{v}_{1 . . \ell}}\left(\hat{\mathbf{s}}, \mathbf{v}_{1 . \ell \ell-1}\right)}-\log \frac{\left.h\right|_{S_{i}^{1} \cup S_{i}^{2}, \mathbf{v}_{1 . . \ell}}\left(\mathbf{v}_{1 . . \ell}\right)}{\left.h\right|_{S_{i}^{1} \cup S_{i}^{2}, \mathbf{v}_{1 . . \ell}}\left(\mathbf{v}_{1 . . \ell-1}\right)}\right) d \mathbf{v}_{1 . . \ell} .
\end{aligned}
$$

Using (18)-(20), this can be further simplified to

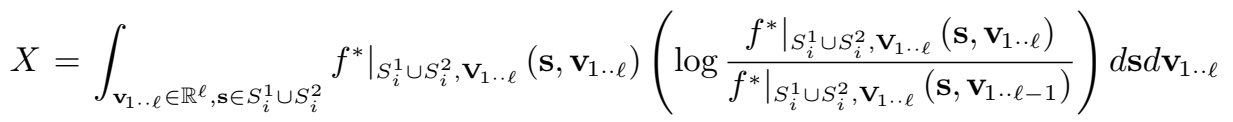

$$
\begin{aligned}
& -\left.\int_{\mathbf{v}_{1} . . \ell \in \mathbb{R}^{\ell}} h\right|_{S_{i}^{1} \cup S_{i}^{2}, \mathbf{v}_{1} . . \ell}\left(\hat{\mathbf{s}}, \mathbf{v}_{1 . . \ell}\right)\left(\log \frac{\left.h\right|_{S_{i}^{1} \cup S_{i}^{2}, \mathbf{v}_{1} . \ell}(\hat{\mathbf{s}}, \mathbf{v})}{\left.h\right|_{S_{i}^{1} \cup S_{i}^{2}, \mathbf{v}_{1} . . \ell}\left(\hat{\mathbf{s}}, \mathbf{v}_{1 . \ell \ell-1}\right)}\right) d \mathbf{v}_{1 . . \ell} \\
& =\mathcal{H}\left(\left(\left.f^{*}\right|_{S_{i}^{1} \cup S_{i}^{2}, \mathbf{V}_{1} \ldots \ell}\right)_{\mathbf{S}, \mathbf{V}_{1} \ldots \ell-1}\right)-\mathcal{H}\left(\left.f^{*}\right|_{S_{i}^{1} \cup S_{i}^{2}, \mathbf{V}_{1} \ldots \ell}\right)-\left[\mathcal{H}\left(\left(\left.f^{*}\right|_{S_{i}^{1} \cup S_{i}^{2}, \mathbf{V}_{1} \ldots \ell}\right)_{\mathbf{V}_{1} \ldots \ell-1}\right)-\mathcal{H}\left(\left.f^{*}\right|_{S_{i}^{1} \cup S_{i}^{2}, \mathbf{V}_{1} \ldots \ell}\right)_{\mathbf{V}_{1} \ldots \ell}\right] \\
& =\left.\mathcal{I}_{f^{*} \mid}\right|_{S_{i}^{1} \cup S_{i}^{2}, \mathbf{V}_{1} . . \ell}\left(\mathbf{S}, \mathbf{V}_{1 . . \ell} \mid \mathbf{V}_{1 . \ell \ell-1}\right) \geq 0 \text {. }
\end{aligned}
$$

Since we have multiple posteriors on $S_{i}^{1} \cup S_{i}^{2}$, it cannot be true that for all $\ell \in A, \mathbf{S}$ and $\mathbf{V}_{1 . . \ell}$ are independent conditional on $\mathbf{V}_{1 \cdot \ell-1}$. Hence, there exists $\ell$ for which the last term is strictly positive. But then $\mathcal{I}_{f^{*}}\left(V_{\ell}, \mathbf{S} \mid \mathbf{V}_{1 \cdot \ell-1}\right)-\mathcal{I}_{h}\left(V_{\ell}, \mathbf{S} \mid \mathbf{V}_{1 \cdot \ell-1}\right)>0$, implying that $h$ is strictly cheaper than $f^{*}$; a contradiction.

\section{Proof of Proposition 1}

According to Lemma 1, an optimal information strategy $f$ necessitates a single posterior for all signals $\mathbf{s} \in S_{i}$. Thus, the revenue function becomes

$$
\begin{aligned}
& \int_{\mathbf{v} \in \mathbb{R}^{n}} \int_{\mathbf{s} \in \mathbb{R}^{n}} U\left(f_{\mathbf{V} \mid \mathbf{s}}\right) f_{\mathbf{S} \mid \mathbf{v}}(d \mathbf{s}) g(d \mathbf{v}) \\
= & \int_{\mathbf{v} \in \mathbb{R}^{n}} \sum_{i=1}^{n} \int_{\mathbf{s} \in S_{i}} U\left(f_{\mathbf{V} \mid \mathbf{s}}\right) f_{\mathbf{S} \mid \mathbf{v}}(d \mathbf{s}) g(d \mathbf{v})=\sum_{i=1}^{n} U\left(f_{\mathbf{V} \mid \mathbf{s} \in S_{i}}\right) \int_{\mathbf{v} \in \mathbb{R}^{n}} \int_{\mathbf{s} \in S_{i}} f_{\mathbf{S} \mid \mathbf{v}}(d \mathbf{s}) g(d \mathbf{v}) \\
= & \sum_{i=1}^{n} U\left(f_{\mathbf{V} \mid \mathbf{s} \in S_{i}}\right) \cdot p_{f}(i)=\sum_{i=1}^{n} \int_{\mathbf{v} \in \mathbb{R}^{n}} v_{i} \cdot f_{\mathbf{V} \mid \mathbf{s} \in S_{i}}(\mathbf{v}) \cdot p_{f}(i) d \mathbf{v} \\
= & \sum_{i=1}^{n} \int_{\mathbf{v} \in \mathbb{R}^{n}} v_{i} \cdot f\left(\mathbf{s} \in S_{i}, \mathbf{v}\right) d \mathbf{v}=\sum_{i=1}^{n} \int_{\mathbf{v} \in \mathbb{R}^{n}} v_{i} \cdot p_{f}(i \mid \mathbf{v}) p(d \mathbf{v})
\end{aligned}
$$

Moreover, define for an optimal information strategy $f$ the random variable $\mathbf{A}_{f}$ that takes the value $i$ with the probability that $i$ is chosen under $f, \operatorname{Pr}\left(\mathbf{A}_{f}=i\right)=p_{f}(i)$. The distribution of $\mathbf{V}$ conditional on $\mathbf{S}$ is the same as the distribution of $\mathbf{V}$ conditional on $\mathbf{A}_{f}$, hence knowing $\mathbf{A}_{f}$ is as informative about $\mathbf{V}$ as knowing $\mathbf{S}$, i.e., $\mathcal{I}_{f^{*}}\left(V_{\ell}, \mathbf{S} \mid \mathbf{V}_{1 . \ell-1}\right)=\mathcal{I}_{f^{*}}\left(V_{\ell}, \mathbf{A}_{f^{*}} \mid \mathbf{V}_{1 . \ell-1}\right)$. Formally, this can be shown as follows: $f_{V_{\ell} \mid i, \mathbf{v}_{1} \ldots \ell-1}=f_{V_{\ell} \mid \mathbf{s}, \mathbf{v}_{1} \ldots \ell-1}$ for $\mathbf{s} \in S_{i}$ implies $\mathcal{H}\left(f_{V_{\ell} \mid \mathbf{s}, \mathbf{v}_{1} \ldots \ell-1}\right)=\mathcal{H}\left(f_{V_{\ell} \mid i, \mathbf{v}_{1} \ldots \ell-1}\right)$ for $\mathbf{s} \in S_{i}$. Thus,

$$
\begin{aligned}
& \mathcal{I}_{f^{*}}\left(V_{\ell}, \mathbf{S} \mid \mathbf{V}_{1 . \ell \ell-1}\right) \\
& =\int_{\mathbf{v}_{1} \ldots \ell-1} f\left(\mathbf{v}_{1 . \ell \ell-1}\right) \mathcal{H}\left(f_{V_{\ell} \mid \mathbf{v}_{1} \ldots \ell-1}\right) d \mathbf{v}_{1 \ldots \ell-1}-\int_{\mathbf{v}_{1} \ldots \ell-1} \sum_{i=1}^{n} \int_{\mathbf{s} \in S_{i}} f(\mathbf{s}) f_{\mathbf{V}_{1 . \ell \ell-1} \mid \mathbf{s}}\left(\mathbf{v}_{1 . \ell-1}\right) \mathcal{H}\left(f_{V_{\ell} \mid \mathbf{s}, \mathbf{v}_{1} \ldots \ell-1}\right) d \mathbf{s} d \mathbf{v}_{1 . \ell-1}
\end{aligned}
$$




$$
\begin{aligned}
& =\int_{\mathbf{v}_{1 \cdot \ell-1}} f\left(\mathbf{v}_{1 \cdot \ell \ell-1}\right) \mathcal{H}\left(f_{V_{\ell} \mid \mathbf{v}_{1 \cdot \ell \ell-1}}\right) d \mathbf{v}_{1 \cdot \ell-1}-\int_{\mathbf{v}_{1} \cdot \ell-1} \sum_{i=1}^{n} p_{f}(i) f_{\mathbf{V}_{1 \cdot \ell-1} \mid i}\left(\mathbf{v}_{1 \cdot \ell-1}\right) \mathcal{H}\left(f_{V_{\ell} \mid i, \mathbf{v}_{1} \cdot \ell-1}\right) d \mathbf{s} d \mathbf{v}_{1 \cdot \ell \ell-1} \\
& =\mathcal{I}_{f^{*}}\left(V_{\ell}, \mathbf{A}_{f^{*}} \mid \mathbf{V}_{1 \cdot \ell-1}\right)
\end{aligned}
$$

The cost of information can now be written as $\hat{c}(f)=\sum_{\ell=1}^{n} \lambda_{\ell} \cdot \mathcal{I}_{f}\left(V_{\ell}, \mathbf{A}_{f} \mid \mathbf{V}_{1 \cdot \cdot \ell-1}\right)$. Using $\mathcal{H}\left(p_{f}\right)=$ $-\sum_{i \in A} p_{f}(i) \log p_{f}(i)$ and $\mathcal{H}\left(p_{f}\left(i \mid \mathbf{v}_{1 . . \ell}\right)\right)=-\sum_{i \in A} p_{f}\left(i \mid \mathbf{v}_{1 \cdot . \ell}\right) \log p_{f}\left(i \mid \mathbf{v}_{1 . . \ell}\right)$, we can write the objective in terms of $\left\{p_{f}\left(i \mid \mathbf{v}_{1 . \ell \ell}\right)\right\}_{i \in A}$. Thus, every optimal information strategy $f$ induces choice probabilities $p_{f}$ that also maximize

$$
\max _{p_{f}} \sum_{i \in \mathcal{A}} \int_{\mathbf{v} \in \mathbb{R}^{n}} v_{i} p_{f}(i \mid \mathbf{v}) p(d \mathbf{v})-\sum_{j \in \mathcal{A}} \lambda_{j} \cdot \mathcal{I}_{p}\left(V_{j}, \mathbf{A}_{f} \mid \mathbf{V}_{1 \cdot j-1}\right) .
$$

On the other hand, let $\left\{p^{*}(i \mid \mathbf{v})\right\}_{i \in A}$ be a collection that solves the problem in the proposition. Select $n$ distinct signals $\left\{\hat{\mathbf{s}}_{i}\right\}_{i \in A}$. Define $f^{*}$ by

$$
f^{*}(\mathbf{s}, \mathbf{v})= \begin{cases}p^{*}(i \mid \mathbf{v}) p(\mathbf{v}), & \text { if } \mathbf{s}=\hat{\mathbf{s}} \\ 0, & \text { otherwise }\end{cases}
$$

Then, $f^{*}$ is an optimal strategy according to (9) and (10). To see this, note that $f^{*}$ is consistent with the priors and induces choice probabilities that maximizes the objective in the proposition. Since every other optimal strategy induces choice probabilities that maximize objective in the proposition, there cannot be a strategy that yields a higher payoff then $f^{*}$.

\section{Proof of Theorem 1}

We already proof the general case allowing for $\lambda_{h+1}=\cdots=\lambda_{n}=\infty$. The parameters $\lambda_{h+1}, \ldots, \lambda_{n}$ appear in the respective mutual information terms (14) in the optimization problem of Proposition 1 . Setting these terms to zero implies $p\left(i \mid \mathbf{v}_{1 \cdot . j-1}\right)=p\left(i \mid \mathbf{v}_{1 \cdot \cdot j}\right)$ for all $i$ and all $j \in\{h+1, \ldots, n\}$. The objective then becomes

$$
\max _{\left\{p\left(i \mid \mathbf{v}_{1 . h}\right)\right\}_{i \in \mathcal{A}}} \sum_{i=1}^{h} \int_{\mathbf{v}_{1 \cdot . h} \in \mathbb{R}^{h}} v_{i} p\left(i \mid \mathbf{v}_{1 \cdot . h}\right) p\left(d \mathbf{v}_{1 \cdot \cdot h}\right)+\sum_{i=1+h}^{n} \int_{\mathbf{v} \in \mathbb{R}^{n}} v_{i} p\left(i \mid \mathbf{v}_{1 \cdot \cdot h}\right) p(d \mathbf{v})-\sum_{j=1}^{h} \lambda_{j} \cdot \mathcal{I}_{p}\left(V_{j}, \mathbf{A} \mid \mathbf{V}_{1 \cdot . j-1}\right)
$$

subject to

$$
\begin{aligned}
p\left(i \mid \mathbf{v}_{1 \cdot h h}\right) & \geq 0 \quad \text { for all } i \in \mathcal{A} \text { and } \mathbf{v}_{1 \cdots h} \in \mathbb{R}^{h} \\
\sum_{i \in \mathcal{A}} p\left(i \mid \mathbf{v}_{1 \cdots h}\right) & =1 .
\end{aligned}
$$

Using

$$
\begin{aligned}
& \int_{\mathbf{v} \in \mathbb{R}^{n}} v_{i} p\left(i \mid \mathbf{v}_{1 \cdot \cdot h}\right) p(\mathbf{v}) d \mathbf{v}=\int_{\mathbf{v}_{1 \cdot h h} \in \mathbb{R}^{h}}\left(\int_{\mathbf{v}_{h+1 \cdot n} \in \mathbb{R}^{n-h}} v_{i} p\left(\mathbf{v}_{1 \cdot h+1} \mid \mathbf{v}_{1 \cdot \cdot h}\right) d \mathbf{v}_{h+1 \cdot \cdot n}\right) p\left(i \mid \mathbf{v}_{1 \cdot \cdot h}\right) p\left(\mathbf{v}_{1 \cdot \cdot h}\right) d \mathbf{v}_{1 \cdot \cdot h} \\
& =\int_{\mathbf{v}_{1 . h} \in \mathbb{R}^{h}} \mathbb{E}_{p_{V_{i} \mid \mathbf{v}_{1} \cdot h h}}\left[v_{i}\right] p\left(i \mid \mathbf{v}_{1 \cdot . h}\right) p\left(d \mathbf{v}_{1 \cdot . h}\right)
\end{aligned}
$$

for $i>h$, and insertion of the equation

$$
\mathcal{I}_{p}\left(V_{j}, \mathbf{A} \mid \mathbf{V}_{1 \cdot \cdot j-1}\right)=-\sum_{i \in \mathcal{A}} \int_{\mathbf{v}_{1 \cdot . j} \in \mathbb{R}^{j}} p\left(i \mid \mathbf{v}_{1 \cdot . j}\right)\left(\log p\left(i \mid \mathbf{v}_{1 \cdot \cdot j-1}\right)-\log p\left(i \mid \mathbf{v}_{1 \cdot \cdot j}\right)\right) p\left(d \mathbf{v}_{1 \cdot . j}\right)
$$


yields the Lagrangian

$$
\begin{aligned}
& \mathfrak{L}\left(\left\{p\left(i \mid \mathbf{v}_{1 . . h}\right)\right\}_{i \in \mathcal{A}}\right)=\sum_{i=1}^{h} \int_{\mathbf{v}_{1 . . h} \in \mathbb{R}^{h}} v_{i} p\left(i \mid \mathbf{v}_{1 . . h}\right) p\left(d \mathbf{v}_{1 . . h}\right)+\sum_{i=h+1}^{n} \int_{\mathbf{v}_{1 . . h} \in \mathbb{R}^{h}} \mathbb{E}_{p_{V_{i} \mid \mathbf{v}_{1 . . h}}}\left[v_{i}\right] p\left(i \mid \mathbf{v}_{1 . . h}\right) p\left(d \mathbf{v}_{1 . . h}\right) \\
& +\sum_{j=1}^{h} \lambda_{j} \cdot \sum_{i \in \mathcal{A}} \int_{\mathbf{v}_{1 . . j} \in \mathbb{R}^{j}} p\left(i \mid \mathbf{v}_{1 . . j}\right)\left(\log p\left(i \mid \mathbf{v}_{1 . \cdot j-1}\right)-\log p\left(i \mid \mathbf{v}_{1 . . j}\right)\right) p\left(d \mathbf{v}_{1 \cdot . j}\right) \\
& +\int_{\mathbf{v}_{1 . . h} \in \mathbb{R}^{h}} \xi_{i}\left(\mathbf{v}_{1 . . h}\right) p\left(i \mid \mathbf{v}_{1 . . h}\right) p\left(d \mathbf{v}_{1 . . h}\right) \\
& -\int_{\mathbf{v}_{1 . h h} \in \mathbb{R}^{h}} \mu\left(\mathbf{v}_{1 \cdots h}\right)\left(\sum_{i=1}^{n} p\left(i \mid \mathbf{v}_{1 . h}\right) p\left(d \mathbf{v}_{1 \cdots h}\right)-1\right) p\left(d \mathbf{v}_{1 \cdots h}\right) .
\end{aligned}
$$

If $p(i)>0$, then $p\left(i \mid \mathbf{v}_{1 . . j}\right)>0$ for $j<h+1$. To see this, suppose there exists a smallest $m$ such that $p\left(i \mid \mathbf{v}_{1 . \cdot m}\right)=0$ for some $\mathbf{v}_{1 \cdot . m}$ and $p\left(i \mid \mathbf{v}_{1 . . k}\right)>0$ for all $k<m$. Then, $p\left(i, \mathbf{v}_{1 \cdot m}\right)=0$, implying $p\left(i, \mathbf{v}_{1 \cdot . k}\right)=0$ and hence $p\left(i \mid \mathbf{v}_{1 . . k}\right)=0$ for all $k>m$ and all $\mathbf{v}_{1 . k}$ with positive measure. Yet, an incremental increase in $p\left(i \mid \mathbf{v}_{1 . \cdot m}\right)$ and setting $p\left(i \mid \mathbf{v}_{1 \cdot . m}\right)=p\left(i \mid \mathbf{v}_{1 . . k}\right)$ for all $k>m$ would reduce the cost of information by

$$
\lim _{p\left(i \mid \mathbf{v}_{1 \cdots m}\right) \backslash 0} \frac{d}{d p\left(i \mid \mathbf{v}_{1 \cdots m}\right)} p\left(i \mid \mathbf{v}_{1 \cdots m}\right)\left(\log p\left(i \mid \mathbf{v}_{1 \cdots m-1}\right)-\log p\left(i \mid \mathbf{v}_{1 \cdots m}\right)\right)=-\infty .
$$

Thus, $p\left(i \mid \mathbf{v}_{1 . . m}\right)=0$ cannot be optimal. Consequently, $\xi_{i}\left(\mathbf{v}_{1 . . h}\right)=0$.

The FOCs w.r.t. $p\left(i \mid \mathbf{v}_{1 . . h}\right)$ are

$$
\begin{aligned}
0= & \int_{\mathbf{v}_{1 . h} \in \mathbb{R}^{h}} v_{i}-\mu\left(\mathbf{v}_{1 . . h}\right) p\left(d \mathbf{v}_{1 . . h}\right)+\lambda_{1} \cdot(\log p(i)+1) \\
& +\sum_{j=1}^{h-1}\left(\lambda_{j+1}-\lambda_{j}\right) \cdot \int_{\mathbf{v}_{1 . . j}}\left(\log p\left(i \mid \mathbf{v}_{1 . . j}\right)+1\right) p\left(d \mathbf{v}_{1 . . j}\right)-\lambda_{h} \cdot \int_{\mathbf{v}_{1 . . h}}\left(\log p\left(i \mid \mathbf{v}_{1 . h}\right)+1\right) p\left(d \mathbf{v}_{1 . . h}\right)
\end{aligned}
$$

for $i \leq h$, while for $i>h$ we obtain

$$
\begin{aligned}
& 0=\int_{\mathbf{v}_{1 . . h} \in \mathbb{R}^{h}} \mathbb{E}_{p_{V_{i} \mid \mathbf{v}_{1 . . h}}}\left[v_{i}\right]-\mu\left(\mathbf{v}_{1 \ldots h}\right) p\left(d \mathbf{v}_{1 . . h}\right)+\lambda_{1} \cdot(\log p(i)+1) \\
& +\sum_{j=1}^{h-1}\left(\lambda_{j+1}-\lambda_{j}\right) \cdot \int_{\mathbf{v}_{1 . . j}}\left(\log p\left(i \mid \mathbf{v}_{1 . . j}\right)+1\right) p\left(d \mathbf{v}_{1 . . j}\right)-\lambda_{h} \cdot \int_{\mathbf{v}_{1 . . h}}\left(\log p\left(i \mid \mathbf{v}_{1 . h h}\right)+1\right) p\left(d \mathbf{v}_{1 . . h}\right)
\end{aligned}
$$

We get

$$
\begin{aligned}
& 0=v_{i}-\mu\left(\mathbf{v}_{1 . . h}\right)+\lambda_{1} \log p(i)+\sum_{j=1}^{h-1}\left(\lambda_{j+1}-\lambda_{j}\right) \cdot \log p\left(i \mid \mathbf{v}_{1 . . j}\right)-\lambda_{h} \log p\left(i \mid \mathbf{v}_{1 . . h}\right) \quad \text { for } i \leq h \text { and } \\
& 0=\mathbb{E}_{p_{V_{i} \mid \mathbf{v}_{1 . . h}}}\left[v_{i}\right]-\mu\left(\mathbf{v}_{1 \cdots h}\right)+\lambda_{1} \log p(i)+\sum_{j=1}^{h-1}\left(\lambda_{j+1}-\lambda_{j}\right) \cdot \log p\left(i \mid \mathbf{v}_{1 . . j}\right)-\lambda_{h} \log p\left(i \mid \mathbf{v}_{1 \ldots h}\right) \quad \text { for } i>h,
\end{aligned}
$$

implying

$$
\begin{array}{ll}
p\left(i \mid \mathbf{v}_{1 . . h}\right)=e^{\frac{v_{i}-\mu\left(\mathbf{v}_{1 . . h}\right)}{\lambda_{h}}} \cdot p(i)^{\frac{\lambda_{1}}{\lambda_{h}}} \cdot \prod_{k=1}^{h-1} p\left(i \mid \mathbf{v}_{1 . . k}\right)^{\frac{\lambda_{k+1}-\lambda_{k}}{\lambda_{h}}} & \text { for } i \leq h \text { and } \\
p\left(i \mid \mathbf{v}_{1 . . h}\right)=e^{\frac{\mathbb{E}_{p}\left[v_{i}\right]-\mu\left(\mathbf{v}_{1 . . h}\right)}{\lambda_{h}}} \cdot p(i)^{\frac{\lambda_{1}}{\lambda_{h}}} \cdot \prod_{k=1}^{h-1} p\left(i \mid \mathbf{v}_{1 . . k}\right)^{\frac{\lambda_{k+1}-\lambda_{k}}{\lambda_{h}}} & \text { for } i>h .
\end{array}
$$

Summing (22) and (23) up over $j \in A$ yields

$1=\sum_{j=1}^{h} e^{\frac{v_{i}-\mu\left(\mathbf{v}_{1} . h\right)}{\lambda_{h}}} \cdot p(i)^{\frac{\lambda_{1}}{\lambda_{h}}} \cdot \prod_{k=1}^{h-1} p\left(i \mid \mathbf{v}_{1 . . k}\right)^{\frac{\lambda_{k+1}-\lambda_{k}}{\lambda_{h}}}+\sum_{j=h+1}^{n} e^{\frac{\mathbb{E}_{p_{i} \mid \mathbf{v}_{1 . . h}}\left[v_{i}\right]-\mu\left(\mathbf{v}_{1 . . h}\right)}{\lambda_{h}}} \cdot p(i)^{\frac{\lambda_{1}}{\lambda_{h}}} \cdot \prod_{k=1}^{h-1} p\left(i \mid \mathbf{v}_{1 . . k}\right)^{\frac{\lambda_{k+1}-\lambda_{k}}{\lambda_{h}}}$ 
Solving for $\mu\left(\mathbf{v}_{1 . . h}\right)$ gives

$\mu\left(\mathbf{v}_{1 . . h}\right)=\lambda_{h} \ln \left(\sum_{j=1}^{h} e^{\frac{v_{i}}{\lambda_{h}}} \cdot p(i)^{\frac{\lambda_{1}}{\lambda_{h}}} \cdot \prod_{k=1}^{h-1} p\left(i \mid \mathbf{v}_{1 . . k}\right)^{\frac{\lambda_{k+1}-\lambda_{k}}{\lambda_{h}}}+\sum_{j=h+1}^{n} e^{\frac{\mathbb{E}_{V_{i} \mid \mathbf{v}_{1 . . h}}\left[v_{i}\right]}{\lambda_{h}}} \cdot p(i)^{\frac{\lambda_{1}}{\lambda_{h}}} \cdot \prod_{k=1}^{h-1} p\left(i \mid \mathbf{v}_{1 . . k}\right)^{\frac{\lambda_{k+1}-\lambda_{k}}{\lambda_{h}}}\right)$

and plugging this into (22) and (23), respectively, yields the desired equations.

\section{Proof of Proposition 2}

Insertion of equation (15) into the objective of Proposition 1 yields

$$
\begin{aligned}
& \sum_{i \in \mathcal{A}} \int_{\mathbf{v} \in \mathbb{R}^{n}} v_{i} p(i \mid \mathbf{v}) p(d \mathbf{v})+\sum_{j=1}^{n-1} \lambda_{j} \cdot\left\{\sum_{i \in A} \int_{\mathbf{v}_{1 \cdot . j} \in \mathbb{R}^{j}} p\left(i \mid \mathbf{v}_{1 \cdot . j}\right)\left(\log p\left(i \mid \mathbf{v}_{1 \cdot \cdot j-1}\right)-\log p\left(i \mid \mathbf{v}_{1 \cdot . j}\right)\right) p\left(d \mathbf{v}_{1 \cdot . j}\right)\right\} \\
& +\lambda_{n} \cdot\left\{\sum_{i \in \mathcal{A}} \int_{\mathbf{v} \in \mathbb{R}^{n}} p(i \mid \mathbf{v})\left(\log p\left(i \mid \mathbf{v}_{1 \cdots n-1}\right)-\log \frac{e^{\frac{v_{i}}{\lambda_{n}}} \cdot p(i)^{\frac{\lambda_{1}}{\lambda_{n}}} \cdot \prod_{k=1}^{n-1} p\left(i \mid \mathbf{v}_{1 . . k}\right)^{\frac{\lambda_{k+1}-\lambda_{k}}{\lambda_{n}}}}{\sum_{j=1}^{n} e^{\frac{v_{j}}{\lambda_{n}}} \cdot p(j)^{\frac{\lambda_{1}}{\lambda_{n}}} \cdot \prod_{k=1}^{n-1} p\left(j \mid \mathbf{v}_{1 . k}\right)^{\frac{\lambda_{k+1}-\lambda_{k}}{\lambda_{n}}}}\right) p(d \mathbf{v})\right\} \\
& =\sum_{i \in \mathcal{A}} \int_{\mathbf{v} \in \mathbb{R}^{n}} v_{i} p(i \mid \mathbf{v}) p(d \mathbf{v})+\sum_{j=1}^{n-1} \lambda_{j} \cdot\left\{\sum_{i \in \mathcal{A}} \int_{\mathbf{v}_{1 . . j} \in \mathbb{R}^{j}} p\left(i \mid \mathbf{v}_{1 \cdot . j}\right)\left(\log p\left(i \mid \mathbf{v}_{1 \cdot . j-1}\right)-\log p\left(i \mid \mathbf{v}_{1 \cdot . j}\right)\right) p\left(d \mathbf{v}_{1 \cdot . j}\right)\right\} \\
& +\lambda_{n} \cdot\left\{\sum_{i \in \mathcal{A}} \int_{\mathbf{v} \in \mathbb{R}^{n}} p(i \mid \mathbf{v}) \log p\left(i \mid \mathbf{v}_{1 . . n-1}\right) p(d \mathbf{v})\right\} \\
& -\left\{\sum_{i \in \mathcal{A}} \int_{\mathbf{v} \in \mathbb{R}^{n}} p(i \mid \mathbf{v})\left(v_{i}+\lambda_{1} \log p(i)+\sum_{k=1}^{n-1} \lambda_{k+1} \log p\left(i \mid \mathbf{v}_{1 . . k}\right)-\sum_{k=1}^{n-1} \lambda_{k} \log p\left(i \mid \mathbf{v}_{1 . . k}\right)\right) p(d \mathbf{v})\right\} \\
& +\lambda_{n} \cdot\left\{\sum_{i \in \mathcal{A}} \int_{\mathbf{v} \in \mathbb{R}^{n}} p(i \mid \mathbf{v})\left(\log \left(\sum_{j=1}^{n} e^{\frac{v_{j}}{\lambda_{n}}} \cdot p(j)^{\frac{\lambda_{1}}{\lambda_{n}}} \cdot \prod_{k=1}^{n-1} p\left(j \mid \mathbf{v}_{1 \cdots k}\right)^{\frac{\lambda_{k+1}-\lambda_{k}}{\lambda_{n}}}\right)\right) p(d \mathbf{v})\right\} \\
& =\lambda_{n} \int_{\mathbf{v}} \log \left(\sum_{j \in \mathcal{A}} e^{\frac{v_{j}}{\lambda_{n}}} \cdot p(j)^{\frac{\lambda_{1}}{\lambda_{n}}} \cdot \prod_{k=1}^{n-1} p\left(j \mid \mathbf{v}_{1 . . k} \frac{\frac{\lambda_{k+1}-\lambda_{k}}{\lambda_{n}}}{n}\right) p(d \mathbf{v})\right. \text {. }
\end{aligned}
$$

\section{Proof of Proposition 3}

For duplicates with $\lambda_{\bar{m}}>\lambda_{m}$, we have $\mathcal{I}_{p}\left(V_{\bar{m}}, \hat{\mathbf{A}} \mid \mathbf{V}_{1 \cdots \bar{m}-1}\right)=0$, i.e., the cost of information $\lambda_{\bar{m}}$ is irrelevant. Moreover, learning about $\bar{m}$ beyond $m$ is not possible, hence $\hat{p}\left(i \mid \mathbf{u}_{1 \cdot \cdots \bar{m}}\right)=\hat{p}\left(i \mid \mathbf{u}_{1 \cdot \cdot \bar{m}-1}\right)$. Thus, the objectives of the problem with and without the duplicate are identical if $\hat{p}\left(\bar{m} \mid \mathbf{u}_{1 . . \ell}\right)=0$. Let the optimal conditional choice probabilities be given by $\hat{p}\left(i \mid \mathbf{u}_{1 . . \ell}\right)$ for $i \in \mathcal{A}$. Inspection of the cost function in (21) clarifies that one can shift conditional choice probability from $m$ to $\bar{m}$ without loosing optimality. In particular, shifting all the probability of $\hat{p}\left(\bar{m} \mid \mathbf{u}_{1 . . \ell}\right)$ to $\hat{p}\left(m \mid \mathbf{u}_{1 . \ell \ell}\right)$ yields an optimal solution that is also a feasible solution of the of the problem without the duplicate. Hence, $p^{*}\left(i \mid \mathbf{v}_{1 . . \ell}\right):=\hat{p}\left(i \mid \mathbf{u}_{1 . . \ell}\right)$ for all $i \in \hat{\mathcal{A}} \backslash\{m, \bar{m}\}$ and $p^{*}\left(m \mid \mathbf{v}_{1 . \ell \ell}\right):=\hat{p}\left(m \mid \mathbf{u}_{1 . \bar{m}}\right)+\hat{p}\left(\bar{m} \mid \mathbf{u}_{1 \cdots \bar{m}-1}\right)$ solves the problem without the duplicate. On the other hand, setting $\hat{p}^{*}\left(i \mid \mathbf{u}_{1 . . \ell}\right):=p\left(i \mid \mathbf{v}_{1 . . \ell}\right)$ for all $i \in \hat{\mathcal{A}} \backslash\{\bar{m}\}$ and $\hat{p}^{*}\left(\bar{m} \mid \mathbf{u}_{1 . . \ell}\right)=0$ yields a solution of the problem with the duplicate whenever $p\left(i \mid \mathbf{v}_{1 . . \ell}\right), i \in \hat{\mathcal{A}} \backslash\{\bar{m}\}$, solves the problem without the duplicate.

\section{Appendix B: General formulae for the limiting scenarios}

In this section, we present the formula for $p(i \mid \mathbf{v})$ for special cases. Using the notation

$$
p(i \mid \mathbf{v})=\frac{q(i \mid \mathbf{v})}{\sum_{j \in A} q(j \mid \mathbf{v})}
$$

it suffices to specify $q(j \mid \mathbf{v})$ for $j \in \mathcal{A}$. 


\section{Identical search cost for some alternatives}

If $\lambda_{h}=\cdots=\lambda_{h+\ell}$, then

$$
q(i \mid \mathbf{v})=e^{\frac{v_{i}}{\lambda_{n}}} \cdot p(i)^{\frac{\lambda_{1}}{\lambda_{n}}} \cdot \prod_{k=1}^{h-1} p\left(i \mid \mathbf{v}_{1 . . k}\right)^{\frac{\lambda_{k+1}-\lambda_{k}}{\lambda_{n}}} \cdots \cdots 1 \cdots \prod_{k=h+\ell}^{n-1} p\left(i \mid \mathbf{v}_{1 . . k}\right)^{\frac{\lambda_{k+1}-\lambda_{k}}{\lambda_{n}}} .
$$

\section{Zero search cost for some alternatives}

Consider the case where $\lambda_{i}=0$ for $i \leq \ell$. Then

$$
q(i \mid \mathbf{v})=e^{\frac{v_{i}}{\lambda_{n}}} \cdot p\left(i \mid \mathbf{v}_{1 . \cdot \ell}\right)^{\frac{\lambda_{\ell+1}}{\lambda_{n}}} \cdot \prod_{k=\ell+1}^{n-1} p\left(i \mid \mathbf{v}_{1 \cdot . k}\right)^{\frac{\lambda_{k+1}-\lambda_{k}}{\lambda_{n}}} .
$$

Note that the DM's conditional choice probabilities are the same as if she knew the state $\mathbf{v}_{1 . . \ell}$ and would not distinguish between $p(i), p\left(i \mid \mathbf{v}_{1}\right), \ldots$, and $p\left(i \mid \mathbf{v}_{1 . . \ell}\right)$ : We come to the same result if we replace $p\left(i \mid \mathbf{v}_{1 . . k}\right)$ by $p\left(i \mid \mathbf{v}_{1 . . \ell}\right)$ for all $k \leq \ell$ in (15).

\section{Infinite search cost for some alternatives}

If $\lambda_{h+1}=\cdots=\lambda_{n}=\infty$, we obtain

$$
q(i \mid \mathbf{v})=e^{\frac{v_{i}}{\lambda_{h}}} \cdot p(i)^{\frac{\lambda_{1}}{\lambda_{h}}} \cdot \prod_{k=1}^{h-1} p\left(i \mid \mathbf{v}_{1 . . k}\right)^{\frac{\lambda_{k+1}-\lambda_{k}}{\lambda_{h}}}
$$

for all $i \leq h$ and

$$
q(i \mid \mathbf{v})=e^{\frac{\bar{v}_{i}}{\lambda_{h}}} \cdot p(i)^{\frac{\lambda_{1}}{\lambda_{h}}} \cdot \prod_{k=1}^{h-1} p\left(i \mid \mathbf{v}_{1 \cdots k}\right)^{\frac{\lambda_{k+1}-\lambda_{k}}{\lambda_{h}}}
$$

for all $i>h$, where $\bar{v}_{i}=\mathbb{E}_{p_{V_{i} \mid \mathrm{v}_{1} \ldots \ell}}\left[v_{i}\right]$.

Zero and infinite search cost for some alternatives

$0=\lambda_{1}=\cdots=\lambda_{\ell}, 0<\lambda_{\ell+1}, \ldots, \lambda_{h}<\infty$, and $\lambda_{h+1}=\cdots=\lambda_{n}=\infty$, gives

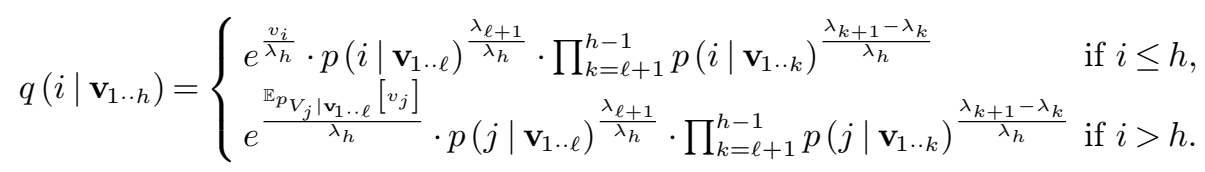

If further $\lambda_{\ell}=\cdots=\lambda_{h}=: \lambda$, this reduces to

$$
q\left(i \mid \mathbf{v}_{1 . . h}\right)= \begin{cases}e^{\frac{v_{i}}{\lambda}} \cdot p\left(i \mid \mathbf{v}_{1 . . \ell}\right), & \text { if } i \leq h, \\ e^{\frac{\mathbb{E}_{p_{V}} \mid \mathbf{v}_{1} . . \ell}{\lambda}} \cdot p\left(i \mid \mathbf{v}_{1} \ldots \ell\right), & \text { if } i>h .\end{cases}
$$




\section{Recent ESMT Working Papers}

ESMT No.

Are level 3 fair values reflected in firm value? Evidence from European banks

Katja Kisseleva, ESMT European School of Management and Technology

Daniela Lorenz, Free University, Berlin

Design for reusability and product reuse under radical innovation

$16-02$

Tamer Boyacl, ESMT European School of Management and Technology

Vedat Verter, Desautels Faculty of Management, McGill University

Michael R. Galbreth, Moore School of Business, University of South Carolina

Pricing when customers have limited attention

Tamer Boyacı, ESMT European School of Management and Technology

Yalçın Akçay, College of Administrative Sciences and Economics, Koç University

Regional state aid control in Europe: A legal and economic assessment

Hans W. Friederiszick, ESMT European School of Management and Technology

Massimo Merola, Bonelli Erede, College of Europe of Bruges

Fund flows inducing mispricing of risk in competitive financial markets

Axel Stahmer, ESMT European School of Management and Technology

LeChatelier-Samuelson principle in games and pass-through of shocks

Alexei Alexandrov, Consumer Financial Protection Bureau

Özlem Bedre-Defolie, ESMT European School of Management and Technology

Contracts as a barrier to entry when buyers are non-pivotal

Özlem Bedre-Defolie, ESMT European School of Management and Technology Gary Biglaiser, University of North Carolina, Chapel Hill 